\title{
WEAK INFINITESIMAL GENERATOR FOR A STOCHASTIC PARTIAL DIFFERENTIAL EQUATION WITH TIME DELAY
}

\author{
MOU-HSIUNG CHANG \\ University of Alabama in Huntsville \\ Department of Mathematical Sciences \\ Huntsville, AL 35899 USA
}

(Received September, 1994; Revised March, 1995)

\begin{abstract}
In this paper, we consider the Markov solution process for a stochastic parabolic differential equation with time delay. Under the Lipschitz condition and boundedness on the drift and diffusion coefficient, properties of the weak infinitesimal generator of the associated Markov operators are established. Actions of the weak infinitesimal generator on the space of quasi-tame functions are also investigated.
\end{abstract}

Key words: Stochastic Partial Differential Equation with Time Delay, Markov Operators, Weak Infinitesimal Generator.

AMS (MOS) subject classifications: $60 \mathrm{H} 10,60 \mathrm{H} 15,35 \mathrm{~K} 60$.

\section{Introduction}

Let $r>0$ and $\ell>0$. Denote the intervals $[-r, 0]$ and $[0, \ell]$ by $J$ and $L$, respectively. Let $C(J)$ be the space of continuous functions $\eta: J \rightarrow \mathbb{R}$, and let $C_{0}(L)$ be the space of continuous functions $\phi: L \rightarrow \mathbb{R}$ such that $\phi(0)=\phi(\ell)=0$. Let $C_{0}(J \times L)$ be the space of continuous functions $\varrho: J \times l \rightarrow \mathbb{R}$ with $\varrho(t, 0)=\varrho(t, \ell)=0$ for each $t \in J$. It is clear that $C(J), C_{0}(L)$, and $C_{0}(J \times L)$ are separable Banach spaces under the uniform topology. Throughout the paper, we shall use the same notation $\|\cdot\|$ for the sup-norms for all three spaces $C(J), C_{0}(L)$, and $C_{0}(J \times L)$, i.e., $\|\eta\|=\sup _{t \in J}|\eta(t)| \quad$ for $\quad \eta \in C(J), \quad\|\phi\|=\sup _{x \in L}|\phi(x)| \quad$ for $\quad \phi \in C_{0}(L) \quad$ and $\|\varrho\|=\sup |\varrho(t, x)|$ for $\varrho \in C_{0}(J \times L)$. If $\varphi:[-r, \infty) \times L \rightarrow \mathbb{R}$ is a continuous function, with $\varphi(t, 0)=\varphi(t, \ell)=0$ for $t \in[-r, \infty)$, then define for each $(t, x) \in \mathbb{R}+\times L, \varphi_{t}(\cdot, x): J \rightarrow \mathbb{R}$ by $\varphi_{t}(\theta, x)=\varphi(t+\theta, x), \quad \theta \in J$. Note that $\varphi_{t}(\cdot, x) \in C(J), \varphi_{t}(\cdot, \cdot) \in C_{0}(J \times L)$, and for each $t \in[-r, \infty), \varphi(t, \cdot) \in C_{0}(L)$. Let $H=L^{2}(L)$, the space of squared integrable functions. $H$ is a separable Hilbert space with the inner product

$$
(\phi, \psi)=\int_{0}^{\ell} \phi(x) \psi(x) d x, \phi, \psi \in H .
$$

Let $|\cdot|:=(\cdot, \cdot)^{1 / 2}$ be its Hilbertian norm. Then $C_{0}(L)$ can be continuously embedded into $H$. For simplicity of the notation we denote the space $C_{0}(J \times L)$ by $C_{0}$ throughout this paper. Let $A=\frac{d^{2}}{d x^{2}}$ be the differential operator defined on the domain $D(A)$, where 


$$
D(A)=\left\{\phi \in H: \phi, \phi^{\prime} \text { are absolutely continuous, } \phi^{\prime \prime} \in H \text {, and } \phi(0)=\phi(\ell)=0\right\} .
$$

The differential operator $A: D(A) \rightarrow H$ defined above has the spectrum $\sigma(A)$ that consists of the simple eigenvalues

$$
\lambda_{n}=\left(\frac{n \pi}{\ell}\right)^{2}, \quad n=1,2,3, \ldots
$$

with the corresponding eigenfunctions

$$
\phi_{n}(x)=\sqrt{\frac{2}{\ell}} \sin \frac{n \pi x}{\ell}, n=1,2,3 \ldots .
$$

Note that $A \phi_{n}=\lambda_{n} \phi_{n}$ and $\left(\phi_{n}, \phi_{m}\right)=\delta_{n, m}$, where $\delta_{n, m}=1$ if $n=m$ and $\delta_{n, m}=0$ if $n \neq m$. Let $(S(t), t \geq 0)$ be the strongly continuous semigroup in $C_{0}(L)$ generated by the infinitesimal generator $A$. In fact (cf. [16]),

$$
S(t) \phi(x)=\int_{0}^{\ell} G(t, x, y) \phi(y) d y, \quad \phi \in H,
$$

where $G(t, x, y)$ is the Green function for the heat equation $\frac{\partial u}{\partial t}=A u$ with homogeneous Dirichlet boundary conditions.

Let $\left(W(t, x),(t, x) \in \mathbb{R}_{+} \times L\right)$ be the standard Brownian sheet defined on a complete probability space $(\Omega, \mathcal{F}, P)$. For each $t \geq 0$, let $(\mathcal{F}(t), t \geq 0)$ be the increasing family of sub- $\sigma$-algebras of subsets of $\Omega$, where $\mathcal{F}(t)=\sigma(W(s, x), 0 \leq s \leq t, x \in L)$. Let $\left(\stackrel{\circ}{W}(t, x),(t, x) \in \mathbb{R}_{+} \times L\right)$ denote the space-time white noise. Formally, one can write $\stackrel{\circ}{W}(t, x)=\frac{\partial^{2}}{\partial t \partial x} W(t, x)$, (see e.g. [16]).

Consider the following stochastic partial functional differential equation and homogeneous Dirichlet boundary conditions:

$$
\left.\begin{array}{c}
\frac{\partial u}{\partial t}(t, x)=\frac{\partial^{2} u}{\partial x^{2}}(t, x)+f\left(x, u_{t}(\cdot, x)\right)+g\left(x, u_{t}(\cdot, x)\right) \stackrel{\circ}{W}(t, x),(t, x) \in(0, \infty) \times(0, \ell) ; \\
u(t, 0)=u(t, \ell)=0, \quad t \geq 0, \\
u(\theta, x)=\eta(\theta, x),(\theta, x) \in J \times L,
\end{array}\right\}
$$

where $f: L \times C(J) \rightarrow \mathbb{R}$ and $g: L \times C(J) \rightarrow \mathbb{R}$ are continuous functions satisfying Assumptions (A1)(A2) to be specified later. Throughout the end of this paper, we define $\widetilde{f}, \widetilde{g}: C(J \times L) \rightarrow C(L)$ by $\tilde{f}(\eta)(x)=f(x, \eta(\cdot, x))$ and $\tilde{g}(\eta)(x)=g(x, \eta(\cdot, x))$ for all $x \in L$, where $C(J \times L)$ and $C(L)$ are spaces of continuous functions on $J \times L$ and $L$, respectively.

Recently, there was a tremendous interest in the deterministic $(g(x, \phi)=0)$ partial functional differential equation due to its applications to mathematical biology (see [2], [5], [10]). The class of equations of this type include the following logistic equation

$$
\frac{\partial u}{\partial t}(t, x)=\frac{\partial^{2} u}{\partial x^{2}}(t, x)+u(t, x)(1-u(t-r, x)) .
$$

When the time delay $r=0$, equation (1.1) becomes the so-called stochastic reaction-diffusion equation (see e.g., [6], [7], [9], [12], [13], [16]) of the following form:

$$
\left.\begin{array}{c}
\frac{\partial u}{\partial t}(t, x)=\frac{\partial^{2} u}{\partial x^{2}}(t, x)+f(x, u(t, x))+g(x, u(t, x)) \stackrel{\circ}{W}(t, x),(t, x) \in(0, \infty) \times(0, \ell) \\
u(t, 0)=u(t, \ell)=0, \quad t \geq 0 \\
u(0, x)=\eta(x), \quad x \in L .
\end{array}\right\}
$$


Equation (1.1) also includes the well-studied stochastic functional differential equations as recorded in [11] and references contained therein. However, stochastic functional differential equations with spatial diffusion such as equation (1.1) have not been studied at all. The main purpose of this paper is to study the Markovian solution of equation (1.1) and its weak infinitesimal generator under conditions to be specified later.

The solution process of equation (1.1) should be interpreted as follows. An $L^{2}\left(\Omega \times \mathbb{R}_{+} \times L\right)$ stochastic process $u=(u(t, x),(t, x) \in[-r, \infty) \times L)$ defined on the probability space $(\Omega, \mathcal{F}, P)$ is said to be a solution process of equation (1.1) with the initial datum $\eta \in C_{0}$ if the following conditions are satisfied:

(i) $u(t, x)=\eta(t, x)$ for $(t, x) \in J \times L$;

(ii) for each $t \geq 0, u(t, x)$ is $\mathcal{F}(t)$-measurable for all $x \in L$;

(iii) $u(t, x)$ is $P$-a.s. continuous in $(t, x) \in \mathbb{R}_{+} \times L$ and satisfies $P$-a.s. the following stochastic integral equation for each $(t, x) \in \mathbb{R}_{+} \times L$;

$$
\begin{gathered}
u(t, x)=\int_{0}^{\ell} G(t, x, y) \eta(0, y) d y+\int_{0}^{t} \int_{0}^{\ell} G(t-s, x, y) f\left(y, u_{s}(\cdot, y)\right) d y d s \\
\quad+\int_{0}^{t} \int_{0}^{\ell} G(t-s, x, y) g\left(y, u_{s}(\cdot, y)\right) W(d y, d s) .
\end{gathered}
$$

Note that the last integral in the RHS of (1.3) should be interpreted as an Ito integral (see e.g., [16]). The uniqueness of the solution process of equation (1.1) is defined in the usual sense (cf., $[16])$.

Throughout the end of this paper, we shall assume that the functions $f, g: L \times C(J) \rightarrow \mathbb{R}$ satisfy the following two conditions:

(A1) There exists a constant $K>0$ such that $|f(x, \xi)| \leq K$ and $|g(x, \xi)| \leq K$ for all $(x, \xi) \in L \times C(J)$

(A2) There exists a constant $k>0$ such that $|f(x, \xi)-f(x, \zeta)| \leq k\|\xi-\zeta\|$ and $\mid g(x, \xi)-$ $g(x, \zeta) \mid \leq k\|\xi-\zeta\|$ for all $(x, \xi) \in L \times C(J)$.

Under assumptions $(A 1)-(A 2)$, it can be shown (see [11], [16]) by the standard Picard iterations that, for each initial datum $\eta \in C_{0}$, equation (1.1) has a unique solution process $u=$ $(u(t, x),(t, x) \in[-r, \infty) \times L)$.

Let $\left(u_{t}(\cdot, \cdot), t \geq 0\right)$ be the $C_{0}$-valued process of equation (1.1), where for each $t \geq 0$, $u_{t}(\theta, x):=u(t+\theta, x ; \eta),(\theta, x) \in J \times L$. For simplicity, we sometimes write the $C_{0}$-valued solution process $u_{t}$ for $u_{t}(\cdot, \cdot)$. To further stress the dependence of the $C_{0}$-valued solution process for equation (1.1) on its initial datum, we let $\left(T^{t}, t \geq 0\right)$ be the family of solution maps of equation (1.1) defined by $T^{t}(\eta)(\theta, x)=u(t+\theta, x ; \eta)$, for $t \geq 0$, and $(\theta, x) \in J \times L$.

It can be shown (cf. [11]) that for each $t>0, T^{t}: C_{0} \rightarrow L^{2}\left(\Omega, C_{0} ; \mathcal{F}(t)\right)$, where $L^{2}\left(\Omega, C_{0} ; \mathscr{F}(t)\right)$ is the space of all $C_{0}$-valued random variables $\Theta$ that are $\mathscr{F}(t)$-measurable and $E\left(\|\Theta\|^{2}\right):=$ $\int_{\Omega}\|\Theta(\omega)\|^{2} d P(\omega)<\infty$, where again $\|\cdot\|$ is the sup-norm for $C_{0}$.

Following the same argument as that of [11], one can show that for each $\eta \in C_{0}$, the $C_{0^{-}}$ valued stochastic process $\left(T^{t}(\eta), t \geq 0\right)$ is a Markov process defined on $(\Omega, \mathcal{F}, P ;(\mathscr{F}(t), t \geq 0))$ with the time-homogeneous Markov transition probabilities $p(\eta, t, \cdot): \mathscr{B}\left(C_{0}\right) \rightarrow \mathbb{R}$, where $\mathscr{B}\left(C_{0}\right)$ is the Borel $\sigma$-algebra of subsets of $C_{0}$ and $p(\eta, t, B)=P\left(T^{t}(\eta) \in B\right), B \in \mathfrak{B}\left(C_{0}\right)$.

The main purpose of this paper is to investigate the weak infinitesimal generator of the $C_{0^{-}}$ valued Markov solution maps $\left(T^{t}(\eta), t \geq 0\right)$ of equation (1.1). The definition of the weak infinitesimal generator $\mathcal{A}$ is explained as follows. 
Let $C_{b}$ be the Banach space of all bounded uniformly continuous functions $\Psi: C_{0} \rightarrow \mathbb{R}$. Let $\left(P^{t}, t \geq 0\right), P^{t}: \mathrm{e}_{b} \rightarrow \mathrm{e}_{b}$, be the semigroup of Markov operators associated with the Markov solution maps $\left(T^{t}, t \geq 0\right)$ defined by $P^{t}(\Psi)(\eta)=E\left[\Psi\left(T^{t}(\eta)\right)\right], \Psi \in \mathrm{C}_{b}, \eta \in C_{0}$ and $t \geq 0$. The weak infinitesimal generator $\mathcal{A}: D(\mathcal{A}) \rightarrow \mathrm{e}_{b}$ of the Markov solution maps $\left(T^{t}, t \geq 0\right)$ is defined by

$$
\begin{gathered}
\mathcal{A}(\Psi)=w_{t \rightarrow 0+} \frac{P^{t}(\Psi)-\Psi}{t}, \text { i.e. } \\
\mathcal{A}(\Psi)=\lim _{t \rightarrow 0+} \frac{\left\langle P^{t}(\Psi), \mu\right\rangle-\langle\Psi, \mu\rangle}{t}, \quad \forall \mu \in \mathrm{C}_{b}^{*},
\end{gathered}
$$

where $D(\mathcal{A})$ is the set of all $\Psi$ for which the above weak limit exists, $\mathrm{C}_{b}^{*}$ (the topological dual space of $\left.\mathrm{C}_{b}\right)$ is the space of all probability measures $\mu$ on the Borel measurable space $\left(C_{0}, \mathscr{B}\left(C_{0}\right)\right)$, and

$$
\langle\Psi, \mu\rangle:=\int_{C_{0}} \Psi(\eta) d \mu(\eta), \Psi \in \mathrm{C}_{b} \text { and } \mu \in \mathrm{C}_{b}^{*} .
$$

Since the main purpose of this paper is to discuss the property of $\mathcal{A}$, the restrictions on the functions $f$ and $g$ have not been pushed to a minimum for the purpose of establishing an existence and uniqueness result. In fact, an existence result can be proved under a lot weaker growth conditions than those in $(A 1)$ such as a linear growth condition (see e.g., [6], [7], [11], and $[16])$.

This paper is organized as follows. The preliminary results that are needed for establishing the main results of this paper are contained in Section 2. In Section 3, the space of quasi-tame functions and properties of the semigroup of shift operators related to $\mathcal{A}$ are investigated. In particular, the action of the infinitesimal generator of the shift operators on a quasi-tame function is illustrated. Finally, the properties of weak infinitesimal generator $\mathcal{A}$ of the semigroup of Markov operators and its action on a quasi-tame function are studied in Sections 4 and 5, respectively.

\section{Preliminary Results}

The Green function $G(t, x, y)$ associated with the heat equation $\frac{\partial u}{\partial t}=\frac{\partial^{2} u}{\partial x^{2}}$ with homogeneous Dirichlet boundary conditions has the following representations (see e.g., [16]):

$$
G(t, x, y)=\sum_{n=1}^{\infty} \chi(\{t \geq 0\}) \exp \left(-\lambda_{n} t\right) \phi_{n}(x) \phi_{n}(y)
$$

where $\chi(B)$ denotes the indicator function of the set $B$; and

$$
G(t, x, y)=\frac{1}{\sqrt{4 \pi t}} \sum_{n=-\infty}^{\infty}\left\{\exp \left[-\frac{(y-x-2 n \ell)^{2}}{4 \pi t}\right]+\exp \left[-\frac{(y+x-2 n \ell)^{2}}{4 \pi t}\right]\right\} .
$$

It is also known (see e.g. [16]) that $G(t, x, y)$ satisfies the following equations:

1. $G(t+s, x, y)=\int_{0}^{\ell} G(t, x, z) G(s, z, y) d z$ for all $s, t \geq 0$ and $x, y \in L$.

2. $\int_{0}^{t} \int_{0}^{\ell} G^{2}(s, x, y) d y d s<\infty$ for all $t \geq 0$ and $x \in L$ and hence $\lim _{t \rightarrow 0} \int_{0}^{t} \int_{0}^{\ell} G^{2}(s, x, y) d y d s$ $=0$ uniformly in $x \in L$. 
3. $\quad \int_{0}^{t} \int_{0}^{\ell} \sup _{(\theta, x) \in J \times L} G^{2}(t+\theta-s, x, y) d y d s \leq \frac{1}{t \ell} \sum_{k=1}^{\infty} \frac{1}{\lambda_{k}}\left(1-e^{-2 \lambda_{k} t}\right)$.

We need the following two lemmas.

Lemma 2.1: Let $T>0$. If $\phi:[0, T] \times L \rightarrow \mathbb{R}$ is a measurable function such that

$$
\int_{0}^{T} \int_{0}^{\ell} G^{2}(s, x, y) \phi^{2}(s, y) d y d s<\infty
$$

then

$$
\begin{gathered}
E\left\{\sup _{(\theta, x) \in J \times L}\left|\int_{0}^{T+\theta} \int_{0}^{\ell} G(T+\theta-s, x, y) \phi(s, y) W(d y, d s)\right|^{2}\right\} \\
\leq \sup _{x \in L} \int_{0}^{T} \int_{0}^{\ell} G^{2}(T-s, x, y) \phi^{2}(s, y) d y d s .
\end{gathered}
$$

Proof: It is clear from [16] that for all $x \in L$,

$$
B(t, x)=\int_{0}^{t} \int_{0}^{\ell} G(t-s, x, y) \phi(s, y) W(d y, d s)
$$

is an $\mathscr{F}(t)$-martingale. Therefore, by the martingale inequality (see e.g. [16]),

$$
\begin{aligned}
\sup _{(\theta, x) \in J \times L} & \left|\int_{0}^{t+\theta} \int_{0}^{\ell} G(t+\theta-s, x, y) \phi(s, y) W(d y, d s)\right|^{2} \\
& \leq \sup _{x \in L} \int_{0}^{t} \int_{0}^{\ell} G^{2}(T-s, x, y) \phi^{2}(s, y) d y d s .
\end{aligned}
$$

Let $\iota: J \times L \rightarrow \mathbb{R}$ be the simple function defined by

$$
\iota(\theta, x)=\left\{\begin{array}{c}
0 \text { if }-r \leq \theta<0, x \in L, \\
1 \text { if } \theta=0, x \in L,
\end{array}\right.
$$

and let $\Lambda$ be the vector space of simple functions of the form $a \iota$ where $a \in \mathbb{R}$. Clearly $C_{0} \cap \Lambda=$ $\{0\}$, where $0 \in C_{0}$ is the zero function. Let us consider the direct sum $C_{0} \oplus \Lambda$ equipped with the norm

$$
\|\eta+q \iota\|=\sup _{(\theta, x) \in J \times L}|\eta(\theta, x)|+|a|, \quad \eta \in C_{0}, \quad a \in \mathbb{R} .
$$

The proof of the following lemma is similar to that of Lemma 3.1 and Lemma 3.2 of [11], and is therefore omitted.

\section{Lemma 2.2:}

1. Let $\alpha \in C_{0}^{*}$. Then $\alpha$ has a unique (continuous) linear extension $\bar{\alpha}: C_{0} \oplus \Lambda \rightarrow \mathbb{R}$ satisfying the following weak continuous property $(W 1)$ : 
(W1) If $\left\{\eta_{k}\right\}_{k=1}^{\infty}$ is a bounded sequence in $C_{0}$ such that $\eta_{k}(\theta, x) \rightarrow \eta(\theta, x)$ as $k \rightarrow \infty$ for all $(\theta, x) \in J \times L$ for some $\eta \in C_{0} \oplus \Lambda$, then $\alpha\left(\eta_{k}\right) \rightarrow \bar{\alpha}(\eta)$ as $k \rightarrow \infty$. The extension map $e: C_{0}^{*} \rightarrow\left(C_{0} \oplus \Lambda\right)^{*}, \alpha \mapsto \bar{\alpha}$ is a linear isometry injective map.

2. Let $\beta: C_{0} \times C_{0} \rightarrow \mathbb{R}$ be a continuous bilinear map. Then $\beta$ has a unique (continuous) bilinear extension $\bar{\beta}:\left(C_{0} \oplus \Lambda\right) \times\left(C_{0} \oplus \Lambda\right) \rightarrow \mathbb{R}$ satisfying the weak continuity property $(W 2)$ :

(W2) If $\left\{\eta_{k}\right\}_{k=1}^{\infty},\left\{\xi_{k}\right\}_{k=1}^{\infty}$ are bounded sequences in $C_{0}$ such that $\eta_{k}(\theta, x) \rightarrow \eta(\theta, x)$ and $\underline{\xi}_{k}(\theta, x) \rightarrow \xi(\theta, x)$ as $k \rightarrow \infty$ for all $(\theta, x) \in J \times L$ for some $\eta, \xi \in C_{0} \oplus \Lambda$, then $\beta\left(\eta_{k}, \xi_{k}\right) \rightarrow$ $\bar{\beta}(\eta, \xi)$ as $k \rightarrow \infty$.

\section{A Semigroup of Shift Operators}

For each $\eta \in C_{0}$, define $\bar{\eta}:[-r, \infty) \times L \rightarrow \mathbb{R}$ by

$$
\bar{\eta}(t, x)=\left\{\begin{array}{c}
\int_{0}^{\ell} G(t, x, y) \eta(0, y) d y \text { if }(t, x) \in \mathbb{R}_{+} \times L, \\
\eta(t, x) \text { if }(t, x) \in J \times L .
\end{array}\right.
$$

Define also the shift operator $S^{t}: \mathrm{e}_{b} \rightarrow \mathrm{e}_{b}$ by setting

$$
S^{t}(\Psi)(\eta)=\Psi\left(\bar{\eta}_{t}\right), \eta \in C_{0}, \quad \Psi \in \mathbb{C}_{b} .
$$

The following proposition gives a canonical characterization for the strong continuity of the Markov operators $\left(P^{t}, t \geq 0\right)$ in terms of the shift operators $\left(S^{t}, t \geq 0\right)$.

Proposition 3.1: The family of shift operators $\left(S^{t}, t \geq 0\right)$ forms a contraction semigroup on $\mathrm{C}_{b}$ such that for each $\eta \in C_{0}, \quad \lim _{t \rightarrow 0+} S^{t}(\Psi)(\eta)=\lim _{t \rightarrow 0+} P^{t}(\Psi)(\eta)=\Psi(\eta)$ for all $\Psi \in \mathrm{C}_{b}$. Furthermore, $\lim _{t \rightarrow 0^{+}} P^{t}(\Psi)(\eta)=\Psi(\eta)$ uniformly $\eta \in C_{0}$ if and only if $\lim _{t \rightarrow 0_{+}} S^{t}(\Psi)(\eta)=\Psi(\eta)$ uniformly in $\eta \in C_{0}$.

Proof: Let $s, t \geq 0, \quad \eta \in C_{0} . \quad$ Then $\left(S^{t} \circ S^{s}\right)(\Psi)(\eta)=\Psi\left(\left(\bar{\eta}_{s}\right)_{t}^{-}\right)$where $\left(\bar{\eta}_{s}\right)_{t}^{-}(\theta, x)=$ $\left(\bar{\eta}_{s}\right)^{-}(t+\theta, x)$. Now,

$$
\left(\bar{\eta}_{s}\right)^{-}(t+\theta, x)=\left\{\begin{array}{c}
\int_{0}^{\ell} G(t+\theta, x, y) \bar{\eta}_{s}(0, y) d y \text { if }(t+\theta, x) \in \mathbb{R}_{+} \times L, \\
\bar{\eta}_{s}(t+\theta, x) \text { if }(t+\theta, x) \in J \times L .
\end{array}\right.
$$

Note that

$$
\begin{gathered}
\int_{0}^{\ell} G(t+\theta, x, y) \bar{\eta}_{s}(0, y) d y \\
=\int_{0}^{\ell} G(t+\theta, x, y)\left(\int_{0}^{\ell} G(s, y, z) \eta(0, z) d z\right) d y \\
=\int_{0}^{\ell} \int_{0}^{\ell} G(t+\theta, x, y) G(s, y, z) \eta(0, z) d y d z \text { (by Fubini's theorem) }
\end{gathered}
$$


since

$$
=\int_{0}^{\ell} G(t+s+\theta, x, z) \eta(0, z) d z=\bar{\eta}_{t+s}(\theta, x), \text { for } t+\theta \geq 0, s \geq 0, x \in L,
$$

$$
\int_{0}^{\ell} G(t, x, y) G(s, y, z) d y=G(t+s, x, z) \text { for } x, z \in L
$$

If $-r \leq t+\theta \leq 0$ and $t+s+\theta \geq 0$, then

$$
\left(\bar{\eta}_{s}\right)^{-}(t+\theta, x)=\bar{\eta}(t+s+\theta, x)=\int_{0}^{\ell} G(t+s+\theta, x, y) \eta(0, y) d y=\bar{\eta}_{t+s}(\theta, x) .
$$

If $\quad-r \leq t+s+\theta \leq 0$, then $\left(\bar{\eta}_{s}\right)_{t}^{-}(\theta, x)=\left(\bar{\eta}_{s}\right)^{-}(t+\theta, x)=\eta(t+s+\theta, x)=\bar{\eta}_{s+t}(\theta, x)$. This proves that

$$
S^{t}\left(S^{s}(\Psi)\right)(\eta)=S^{t+s}(\Psi)(\eta), \text { i.e. } S^{t} \circ S^{s}=S^{t+s} \text { for all } s, t \geq 0
$$

Therefore $\left(S^{t}, t \geq 0\right)$ forms a semigroup of shift operators from $\mathcal{C}_{b}$ into $\mathcal{C}_{b}$. To show that $S^{t}$ : $e_{b} \rightarrow e_{b}$ is a contraction, we consider

$$
\left\|S^{t}\right\| \sup _{\Psi \in \mathrm{e}_{b}}\left\|S^{t}(\Psi)\right\| \mathrm{e}_{b} /\|\Psi\| \mathrm{e}_{b}
$$

Now,

$$
\begin{gathered}
\left\|S^{t}(\Psi)\right\| \mathrm{e}_{b}=\sup _{\eta \in C_{0}}\left\|S^{t}(\Psi)(\eta)\right\| /\|\eta\| \\
=\sup _{\eta \in C_{0}}\left\|\Psi\left(\bar{\eta}_{t}\right)\right\| /\|\eta\| \\
=\sup _{\eta \in C_{0}}\|\Psi\|\left\|\bar{\eta}_{t}\right\| /\|\eta\| .
\end{gathered}
$$

Note that $\left\|\bar{\eta}_{t}\right\| \leq\|\eta\|$. Thus for each $t \geq 0,\left\|S^{t}\right\| \leq 1$. This shows that $\left(S^{t}, t \geq 0\right)$ is a family of contraction maps from $e_{b}$ into $e_{b}$.

Since for $\theta=0, \lim _{t \rightarrow 0+} \bar{\eta}_{t}(\theta, x)=\lim _{t \rightarrow 0+} \int_{0}^{\ell} G(t, x, y) \eta(0, y) d y=\eta(0, x)$, and for $\theta<0, \lim _{t \rightarrow 0+}$ $\eta_{t}(\theta, x)=\lim _{t \rightarrow 0+} \bar{\eta}(t+\theta, x)=\lim _{t \rightarrow 0+} \eta(t+\theta, x)=\eta(\theta, x)$, i.e., $\quad \lim _{t \rightarrow 0+} \bar{\eta}_{t}=\eta$ it is clear that $\lim _{t \rightarrow 0+}$ $S^{t}(\Psi)(\eta)=\Psi(\eta)$ for each $\Psi \in \mathrm{C}_{b}, \eta \in C_{0}$. Also by sample paths continuity of the trajectory $\left(T^{t}(\eta), t \geq 0\right)$ of equation (1.1) together with the dominated convergence theorem, one obtains

$$
\begin{gathered}
\lim _{t \rightarrow 0+} P^{t}(\Psi)(\eta)=\lim _{t \rightarrow 0+} \int_{\Omega} \Psi\left(T^{t}(\eta)(\omega)\right) d P(\omega) \\
=\int_{\Omega} \Psi\left(\lim _{t \rightarrow 0+} T^{t}(\eta)(\omega)\right) d P(\omega) \\
=\int_{\Omega} \Psi(\eta) d P(\omega)=\Psi(\eta)
\end{gathered}
$$

for each $\Psi \in \mathrm{C}_{b}$ and $\eta \in C_{0}$.

To prove the second part of the proposition, note that $|f(x, \phi)| \leq K$ and $|g(x, \phi)| \leq K$ for 
all $(x, \phi) \in L \times C(J)$. Then for each $t>0,(\theta, x) \in J \times L$ and $P$-a.s., we have

$$
\begin{aligned}
& T^{t}(\eta)(\theta, x)-\bar{\eta}_{t}(\theta, x)= \\
& \left\{\begin{array}{cc}
\int_{0}^{t+\theta} \int_{0}^{\ell} G(t+\theta-s, x, y) f\left(y, T^{s}(\eta)(\cdot, y)\right) d y d s & \\
+\int_{0}^{t+\theta} \int_{0}^{\ell} G(t+\theta-s, x, y) g\left(y, T^{s}(\eta)(\cdot, y)\right) W(d y, d s) & \text { if } t+\theta>0 \\
0 & \text { if } t+\theta \leq 0 .
\end{array}\right.
\end{aligned}
$$

This shows that

$$
\begin{gathered}
E\left\{\sup _{(\theta, x) \in J \times L}\left|T^{t}(\eta)(\theta, x)-\bar{\eta}_{t}(\theta, x)\right|^{2}\right\} \\
\leq 2 E\left\{\sup _{(\theta, x) \in J \times L}\left|\int_{0}^{t+\theta} \int_{0}^{\ell} G(t+\theta-s, x, y) f\left(y, T^{s}(\eta)(\cdot, y)\right) d y d s\right|^{2}\right\} \\
+2 E\left\{\sup _{(\theta, x) \in J \times L}\left|\int_{0}^{t+\theta} \int_{0}^{\ell} G(t+\theta-s, x, y) g\left(y, T^{s}(\eta)(\cdot, y)\right) W(d y, d s)\right|^{2}\right\} \\
\leq 2 E\left\{\operatorname { s u p } _ { ( \theta , x ) \in J \times L } \left[\left(\int_{0}^{t+\theta} \int_{0}^{\ell} G^{2}(t+\theta-s, x, y) d y d s\right)\right.\right. \\
\times \int_{\left.\left.\sup _{(\theta, x) \in J \times L} \int_{0}^{t+\theta} \int_{0}^{\ell} \int_{0}^{\ell}\left(y, T^{s}(\eta)(\cdot, y)\right) d y d s\right)\right]}^{t+\theta} \int_{0}^{\ell}\left[G^{2}(t+\theta-s, x, y) g^{2}\left(y, T^{s}(\eta)(\cdot, y)\right)\right] d y d s
\end{gathered}
$$

(by Lemma 2.1)

$$
\leq 2 \sup _{x \in L}\left(\int_{0}^{t} \int_{0}^{\ell} G^{2}(t-s, x, y) d y d s\right) \cdot K^{2}(\ell t+1) \rightarrow 0 \text { as } t \rightarrow 0+.
$$

Therefore, $E\left\|T^{t}(\eta)-\bar{\eta}_{t}\right\|^{2} \rightarrow 0$, uniformly in $\eta$ as $t \rightarrow 0+$. This proves that if $\Psi \in \mathcal{C}_{b}$ then $\lim _{t \rightarrow 0+} E\left[\Psi\left(T^{t}(\eta)\right)-\Psi\left(\bar{\eta}_{t}\right)\right]=0$ uniformly in $\eta \in C_{0}$. So $\lim _{t \rightarrow 0+}\left\{P^{t}(\Psi)(\eta)-S^{t}(\Psi)(\eta)\right\}=0$ uniformly in $\eta \in C_{0}$.

Finally, writing $P^{t}(\Psi)(\eta)-\Psi(\eta)=\left[P^{t}(\Psi)(\eta)-S^{t}(\Psi)(\eta)\right]+\left[S^{t}(\Psi)(\eta)-\Psi(\eta)\right]$, the second assertion of the theorem is now obvious.

Let $\mathrm{C}_{b}^{0} \subset \mathcal{C}_{b}$ be the set of all $\Psi \in \mathrm{C}_{b}$ such that $\lim _{t \rightarrow 0+} P^{t}(\Psi)=\Psi\left(=\lim _{t \rightarrow 0+} S^{t}(\Psi)\right)$ in $\mathcal{C}_{b}$. Then $e_{b}^{0}$ is a closed linear subalgebra of $e_{b}$ which is invariant under the semigroups $\left(P^{t}, t \geq 0\right)$, and 
$\left(S^{t}, t \geq 0\right)$. Both $\left(P^{t}, t \geq 0\right)$ and $\left(S^{t}, t \geq 0\right)$ are strongly continuous semigroups on $\mathrm{C}_{b}^{0}$.

Let $D(\varphi) \subset \mathcal{C}_{b}^{0}$ be the domain of the weak infinitesimal generator $\varphi$ for the semigroup of shift operators $\left(S^{t}, t \geq 0\right)$ defined in $(3.1)$, where $\varphi: D(\varphi) \rightarrow \mathrm{C}_{b}$ is defined by

$$
\varphi(\Psi)(\eta)=\lim _{t \rightarrow 0+} \frac{1}{t}\left[S^{t}(\Psi)(\eta)-\Psi(\eta)\right]
$$

In the following, we investigate the infinitesimal generator $\varphi$ as well as a space of functions that lie within $D(\varphi)$.

Definition 3.2: A function $\Psi: C_{0} \rightarrow \mathbb{R}$ is said to be a tame function if there exists a finite set of points $\left(\theta_{1}, x_{1}\right),\left(\theta_{2}, x_{2}\right), \ldots,\left(\theta_{k}, x_{k}\right)$ in $J \times L$ and a $C^{\infty}$-bounded function $\alpha: \mathbb{R}^{k} \rightarrow \mathbb{R}$ such that $\Psi(\eta)=\alpha\left(\eta\left(\theta_{1}, x_{1}\right), \ldots, \eta\left(\theta_{k}, x_{k}\right)\right)$ for all $\eta \in C_{0}$. The above representation of $\Psi$ is called minimal if for any projection $p: \mathbb{R}^{k} \rightarrow \mathbb{R}^{k-1}$, there is no function $\beta: \mathbb{R}^{k-1} \rightarrow \mathbb{R}$ with $\alpha=\beta \circ p$; in other words, no partial derivative $D_{j} \alpha$ of $\alpha$ with respect to its $j$ th variable, $j=1,2, \ldots, k$ vanishes identically.

Regarding tame functions, we make the following observations (see e.g. [11]):

(a) Each tame function admits a unique minimal representation.

(b) The set $\mathbb{T}$ of all tame functions on $C_{0}$ is a weakly dense subalgebra of $C_{b}$, invariant under the shift semigroup $\left\{S^{t}, t \geq 0\right\}$ and generating $\mathscr{B}\left(C_{0}\right)$.

(c) If $\Psi \in \mathbb{T}$ has a minimal representation $\Psi(\eta)=\alpha\left(\eta\left(\theta_{1}, x_{1}\right), \ldots, \eta\left(\theta_{k}, x_{k}\right)\right), \eta \in C_{0}$ where $k \geq 2$, then $\Psi \notin \mathrm{C}_{b}^{0}$, where $\mathrm{C}_{b}^{0}$ is the set of all $\Psi \in \mathrm{C}_{b}$ such that $\lim _{t \rightarrow 0+} P^{t}(\Psi)=\Psi$ $\left(=\lim _{t \rightarrow 0+} S^{t}(\Psi)\right)$ in $\mathrm{C}_{b}$.

Since $\mathbb{T} \notin \mathrm{C}_{b}^{0}$, we need to study the set of quasi-tame functions which are defined as follows.

Definition 3.3: A function $\Psi: C_{0} \rightarrow \mathbb{R}$ is said to be quasi-tame if there exists an integer $k>0$ such that

$$
\Psi(\eta)=h\left(\int_{r}^{0} \int_{0}^{\ell} \alpha_{1}(\eta(\theta, x)) \beta_{1}(\theta, x) d x d \theta, \ldots, \int_{-r}^{0} \int_{0}^{\ell} \alpha_{k-1}(\eta(\theta, x)) \beta_{k-1}(\theta, x) d x d \theta, \int_{0}^{\ell} \eta(0, x) d x\right)
$$

for all $\eta \in C_{0}$, where

(i) $\quad h: \mathbb{R}^{k} \rightarrow \mathbb{R}$ is $C^{\infty}$-bounded.

(ii) $\quad \alpha_{i}: \mathbb{R} \rightarrow \mathbb{R}, i=1,2, \ldots, k-1$, is $C^{\infty}$-bounded.

(iii) $\beta_{i}: J \times L \rightarrow \mathbb{R}, i=1,2, \ldots, k-1$, is continuous in $(\theta, x) \in J \times L$ and piecewise $C^{1}$ in $\theta$ for each $x$ and $\frac{\partial \beta_{i}}{\partial \theta}$ is absolutely integrable over $J$.

The set of all quasi-tame functions will be denoted by $\mathbb{Q T}$.

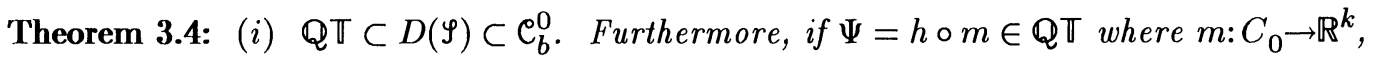

$$
m(\eta)=\left(\int_{-r}^{0} \int_{0}^{\ell} \alpha_{1}(\eta(\theta, x)) \beta_{1}(\theta, x) d x d \theta, \ldots, \int_{-r}^{0} \int_{0}^{\ell} \alpha_{k-1}(\eta(\theta, x)) \beta_{k-1}(\theta, x) d x d \theta, \int_{0}^{\ell} \eta(0, x) d x\right),
$$

$h: \mathbb{R} \rightarrow \mathbb{R}, \alpha_{i}: \mathbb{R} \rightarrow \mathbb{R}$, and $\beta_{i}: J \times L \rightarrow \mathbb{R}, i=1,2, \ldots, k-1$ are as in Definition 3.3 , then

$$
\begin{gathered}
\varphi(\Psi)(\eta):=\lim _{t \rightarrow 0+} \frac{1}{t}\left(S^{t}(\Psi)(\eta)-\Psi(\eta)\right) \\
=\sum_{i=1}^{k-1} D_{i} h(m(\eta)) \cdot\left[-\int_{-r}^{0} \int_{0}^{\ell} \alpha_{i}(\eta(\theta, x)) \frac{\partial \beta_{i}(\theta, x)}{\partial \theta} d x d \theta\right.
\end{gathered}
$$




$$
\begin{gathered}
\left.+\int_{0}^{\ell} \alpha_{i}\left(\int_{0}^{\ell} \eta(0, y) d y\right) \beta_{i}(0, x) d x-\int_{0}^{\ell} \alpha_{i}(\eta(-r, x)) \beta_{i}(-r, x) d x\right] \\
+D_{k} h(m(\eta)) \int_{0}^{\ell} A \eta(0, x) d x
\end{gathered}
$$

where $D_{i} h$ denotes the partial derivative of $h\left(y_{1}, y_{2}, \ldots, y_{k}\right)$ with respect to $y_{i}$, and $A \eta(0, x)=$ $\frac{d^{2}}{d x^{2}} \eta(0, x)$.

(ii) $\mathbb{Q T}$ is invariant under the semigroup $\left(S^{t}, t \geq 0\right)$, i.e., $S^{t}: \mathbb{Q T} \rightarrow \mathbb{Q T}$ for all $t \geq 0$.

(iii) $\mathbb{Q} \mathbb{T}$ is a weakly dense subalgebra of $\mathfrak{C}_{b}$ generating $\mathfrak{B}\left(C_{0}\right)$.

Proof: In proving statements $(i)$ and $(i i)$ of the theorem, we shall assume for the sake of simplicity that $\Psi=h \circ m$, where $h: \mathbb{R}^{2} \rightarrow \mathbb{R}$ is $C^{\infty}$-bounded and

$$
m(\eta)=\left(\int_{-r}^{0} \int_{0}^{\ell} \alpha(\eta(\theta, x)) \beta(\theta, x) d x d \theta, \int_{0}^{\ell} \eta(0, x) d x\right)
$$

for some $C^{\infty}$-bounded map $\alpha: \mathbb{R} \rightarrow \mathbb{R}$ and $\beta(\theta, x), \beta: J \times L \rightarrow \mathbb{R}$, is continuous and piecewise $C^{1}$ in $\Theta$ for each $x \in L$.

Let $t>0$ and consider the expression

$$
\frac{1}{t}\left[\Psi\left(\bar{\eta}_{t}\right)-\Psi(\eta)\right]=\frac{1}{t}\left[h\left(m\left(\bar{\eta}_{t}\right)\right)-h(m(\eta))\right]=\int_{0}^{1} D h\left(y_{\lambda}(t)\right) \cdot\left\{\frac{1}{t}\left[m\left(\bar{\eta}_{t}\right)-m(\eta)\right]\right\} d \lambda,
$$

where $y_{\lambda}(t)=\lambda m\left(\bar{\eta}_{t}\right)+(1-\lambda) m(\eta), 0 \leq \lambda \leq 1$, and

$$
\operatorname{Dh}\left(y_{1}, y_{2}\right)=\left(\begin{array}{c}
\frac{\partial h}{\partial y_{1}}\left(y_{1}, y_{2}\right) \\
\frac{\partial h}{\partial y_{2}}\left(y_{1}, y_{2}\right)
\end{array}\right)
$$

Consider $\frac{1}{t}\left[m\left(\bar{\eta}_{t}\right)-m(\eta)\right]=\frac{1}{t}(I, I I)$, where for $0<t<r$,

$$
\begin{gathered}
\frac{1}{t} I:=\frac{1}{t}\left[\int_{-r}^{0} \int_{0}^{\ell} \alpha\left(\bar{\eta}_{t}(\theta, x)\right) \beta(\theta, x) d x d \theta-\int_{-r}^{0} \int_{0}^{\ell} \alpha(\eta(\theta, x)) \beta(\theta, x) d x d \theta\right] \\
=\frac{1}{t} \int_{-r}^{-t} \int_{0}^{\ell} \alpha(\eta(t+\theta, x)) \beta(\theta, x) d x d \theta
\end{gathered}
$$

$$
+\frac{1}{t} \int_{-t}^{0} \int_{0}^{\ell} \alpha\left(\int_{0}^{\ell} G(t+\theta, x, y) \eta(0, y) d y\right) \beta(\theta, x) d x d \theta-\frac{1}{t} \int_{-r}^{0} \int_{0}^{\ell} \alpha(\eta(\theta, x)) \beta(\theta, x) d x d \theta
$$

$=\frac{1}{t} \int_{t-r}^{0} \int_{0}^{\ell} \alpha(\eta(\theta, x))(\beta(\theta-t, x)-\beta(\theta, x)) d x d \theta+\frac{1}{t} \int_{-t}^{0} \int_{0}^{\ell} \alpha\left(\int_{0}^{\ell} G(t+\theta, x, y) \eta(0, y) d y\right) \beta(\theta, x) d x d \theta$ 


$$
-\frac{1}{t} \int_{-r}^{t-r} \int_{0}^{\ell} \alpha(\eta(\theta, x)) \beta(\theta, x) d x d \theta .
$$

Since $\alpha: \mathbb{R} \rightarrow \mathbb{R}$ is bounded and $\beta: J \times L \rightarrow \mathbb{R}$ is continuous and piecewise $C^{1}$ and $\theta$ for each $x$, we have

$$
\begin{gathered}
\lim _{t \rightarrow 0+} \frac{1}{t} I=-\int_{-r}^{0} \int_{0}^{\ell} \alpha(\eta(\theta, x)) \frac{\partial \beta}{\partial \theta}(\theta, x) d x d \theta+\int_{0}^{\ell} \alpha\left(\int_{0}^{\ell} \eta(0, y) d y\right) \beta(0, x) d x \\
=\int_{0}^{\ell} \alpha(\eta(-r, x)) \beta(-r, x) d x .
\end{gathered}
$$

Note that

$$
\begin{gathered}
\lim _{t \rightarrow 0+} \frac{1}{t} I I=\lim _{t \rightarrow 0+} \frac{1}{t} \int_{0}^{\ell}\left(\bar{\eta}_{t}(0, x)-\eta(0, x)\right) d x \\
=\lim _{t \rightarrow 0+} \frac{1}{t} \int_{0}^{\ell}(S(t) \eta(0, x)-\eta(0, x)) d x=\int_{0}^{\ell} A \eta(0, x) d x
\end{gathered}
$$

since $S(t) \eta(0, x)$ solves the following heat equation

$$
\left\{\begin{array}{c}
\frac{\partial v}{\partial t}=\frac{\partial^{2} v}{\partial x^{2}}(x),(t, x) \in(0, \infty) \times(0, \ell), \\
v(t, 0)=v(t, \ell)=0, t \geq 0 \\
v(0, x)=\eta(0, x), x \in L
\end{array}\right.
$$

and the differential operator $A$ is the infinitesimal generator of the semigroup $(S(t), t \geq 0)$. This shows that

$$
\begin{gathered}
\lim _{t \rightarrow 0} \frac{1}{t}\left[m\left(\bar{\eta}_{t}\right)-m(\eta)\right]=\lim _{t \rightarrow 0} \frac{1}{t}(I, I I) \\
=\left(-\int_{-r}^{0} \int_{0}^{\ell} \alpha(\eta(\theta, x)) \frac{\partial \beta}{\partial \theta}(\theta, x) d x d \theta+\int_{0}^{\ell} \alpha\left(\int_{0}^{\ell} \eta(0, y) d y\right) \beta(0, x) d x-\int_{0}^{\ell} \alpha((\eta(-r, x)) \beta(-r, x) d x\right. \\
\left.\int_{0}^{\ell} A \eta(0, x) d x\right) .
\end{gathered}
$$

By the continuity of $D h\left(y_{1}, y_{2}\right)$, we also have $\lim _{t \rightarrow 0+} D h\left(y_{\lambda}(t)\right)=D h(m(\eta))$. Therefore,

$$
\begin{gathered}
\varphi(\Psi)(\eta):=\lim _{t \rightarrow 0+} \frac{S^{t}(\Psi)(\eta)-\Psi(\eta)}{t} \\
=D_{1} h(m(\eta))\left[-\int_{-r}^{0} \int_{0}^{\ell} \alpha(\eta(\theta, x)) \beta_{\theta}(\theta, x) d x d \theta+\int_{0}^{\ell} \alpha\left(\int_{0}^{\ell} \eta(0, y) d y\right) \beta(0, x) d x\right. \\
\left.-\int_{0}^{\ell} \alpha(\eta(-r, x)) \beta(-r, x) d x\right]+D_{2} h(m(\eta)) \int_{0}^{\ell} A \eta(0, x) d x,
\end{gathered}
$$


where $D_{1} h$ and $D_{2} h$ denote the partial derivatives of $h$ with respect to its first and second variable, respectively, and $A \eta(0, x)=\frac{d^{2}}{d x^{2}} \eta(0, x)$.

(ii) For $\mathbb{Q} \mathbb{T}$ to be invariant under $\left\{S^{t}, t \geq 0\right\}$ it is sufficient to prove that it is invariant under $\left(S^{t}, 0 \leq t \leq r\right)$ due to the semigroup property. Let $\Psi \in \mathrm{C}_{b}$ be defined as $\Psi(\eta)=h \circ m(\eta)$, where $h: \mathbb{R}^{2} \rightarrow \mathbb{R}$ and $m: C_{0} \rightarrow \mathbb{R}^{2}$ are as above. Then,

$$
S^{t}(\Psi)(\eta)=\left(\int_{-r}^{0} \int_{0}^{\ell} \alpha\left(\bar{\eta}_{t}(\theta, x)\right) \beta(\theta, x) d x d \theta, \int_{0}^{\ell} \bar{\eta}_{t}(0, x) d x\right),
$$

where

$$
\int_{0}^{\ell} \bar{\eta}_{t}(0, x) d x=\int_{0}^{\ell} \int_{0}^{\ell} G(t, x, y) \eta(0, y) d y d x=\int_{0}^{\ell} \eta(0, y) d y .
$$

Now,

$$
\begin{gathered}
\int_{-r}^{0} \int_{0}^{\ell} \alpha\left(\bar{\eta}_{t}(\theta, x)\right) \beta(\theta, x) d x d \theta=\int_{-r}^{-t} \int_{0}^{\ell} \alpha(\eta(t+\theta, x)) \beta(\theta, x) d x d x \\
+\int_{-t}^{0} \int_{0}^{\ell} \alpha(S(t+\theta) \eta(0, x)) \beta(\theta, x) d \theta d x \\
=\int_{t-r}^{0} \int_{0}^{\ell} \alpha(\eta(s, x)) \beta(s-t, x) d x d s+\int_{-t}^{0} \int_{0}^{\ell} \alpha\left(\int_{0}^{\ell} G(t+\theta, x, y) \eta(0, x) d y\right) \beta(\theta, x) d \theta d x .
\end{gathered}
$$

This shows that $Q \mathbb{T}$ is invariant under $\left(S^{t}, 0 \leq t \leq r\right)$.

(iii) Finally, we prove that $\mathbb{Q} \mathbb{T}$ generates the Borel subsets of $C_{0}, \mathfrak{B}\left(C_{0}\right)$. For this purpose it is enough to show that $\sigma(\mathbb{Q} \mathbb{T})$, the $\sigma$-algebra generated by $\mathbb{Q T}$, coincides with one generated by the family of evaluations

$$
\begin{gathered}
\rho\left(\left(\theta_{1}, x_{1}\right), \ldots,\left(\theta_{k}, x_{k}\right)\right): C_{0} \rightarrow \mathbb{R}^{k} \\
\rho\left(\left(\theta_{1}, x_{1}\right), \ldots,\left(\theta_{k}, x_{k}\right)\right) \eta=\left(\eta\left(\theta_{1}, x_{1}\right), \eta\left(\theta_{2}, x_{2}\right), \ldots, \eta\left(\theta_{k}, x_{k}\right)\right),
\end{gathered}
$$

where $\left(\theta_{i}, x_{i}\right) \in J \times L, i=1,2, \ldots, k, k=1,2, \ldots$, and $\eta \in C_{0}$. But this is again generated by sets of the form $\{\eta: \eta(\theta, x) \in A\}$ where $A \subseteq \mathbb{R}$ is a closed set. Now $A=\bigcap_{n=1}^{\infty} I_{n}^{c}$, where $I_{n}^{c}$ is the complement of the open interval $I_{n}=\left(a_{n}, b_{n}\right)$. It is sufficient to show that $\left\{\eta \in C_{0}: \eta(\bar{\theta}, \bar{x}) \in I^{c}\right\} \in \sigma(\mathbb{Q} \mathbb{T})$ for any $(\theta, \bar{x}) \in J \times L$. Suppose $I=(y-b, y+b)$ for $y \in \mathbb{R}$ and $b \geq 1$. Let $\bar{I}_{\rho}=\left[y-b+\frac{1}{\rho}, y+b-\frac{1}{\rho}\right]$ and $U_{\rho}=\bar{I}_{\rho}^{c}$. Define a sequence of functions $\alpha^{m}: \mathbb{R} \rightarrow \mathbb{R}$ by

where

$$
\alpha^{m}(y)=\delta_{m}(y) y \text { for } y \in \mathbb{R},
$$

$$
\delta_{m}(y)=\left\{\begin{array}{c}
1, \text { for }|y| \leq m, \\
0, \text { for }|y| \geq m+1, \\
-y+m+1, \text { for } m<y<m+1, \\
y+m+1, \text { for }-m-1<y<-m,
\end{array}\right.
$$


and a sequence of piecewise linear continuous functions $\beta_{(\bar{\theta}, \bar{x})}^{m}: J \times L \rightarrow \mathbb{R}$ by

$$
\beta_{(\bar{\theta}, \bar{x})}^{m}(\theta, x)=\left\{\begin{array}{c}
1 \text { for }(\theta, x)=(\bar{\theta}, \bar{x}), \\
0 \text { for } \sqrt{(\theta-\bar{\theta})^{2}+(x-\bar{x})^{2}} \geq \frac{1}{m}, \\
\text { linear for } 0<\sqrt{(\theta-\bar{\theta})^{2}+(x-\bar{x})^{2}}<\frac{1}{m} .
\end{array}\right.
$$

Let $\Psi_{m}: C_{0} \rightarrow \mathbb{R}$ be defined by

$$
\Psi_{m}(\eta)=\int_{-r}^{0} \int_{0}^{\ell} \alpha^{m}(\eta(\theta, x)) \beta_{(\bar{\theta}, \bar{x})}^{m}(\theta, x) d x d \theta .
$$

It is clear that $\Psi_{m} \in \mathbb{Q} \mathbb{T}$ and $\lim _{m \rightarrow \infty} \Psi_{m}(\eta)=\eta(\bar{\theta}, \bar{x})$. We claim that $\left\{\eta \in C_{0}: \eta(\bar{\theta}, \bar{x}) \in I^{c}\right\}=\bigcap_{\rho=1}^{\infty}$ $\liminf _{m \rightarrow \infty}\left\{\eta \in C_{0}: \Psi_{m}(\eta) \in U_{\rho}\right\}$. To prove this, let $\eta \in C_{0}$ be such that $\eta(\bar{\theta}, \bar{x}) \in I^{c}$. Then $\eta(\bar{\theta}, \bar{x}) \in U_{\rho}$ for all $m \geq \bar{m}$. Hence, for each $\rho \geq 1, \eta \in \liminf _{m \rightarrow \infty}\left\{\eta \in C_{0}: \Psi_{m}(\eta) \in U_{\rho}\right\}$, i.e., $\eta \in \bigcap_{\rho=1}^{\infty} \liminf _{m \rightarrow \infty}\left\{\eta \in C_{0}: \Psi_{m}(\eta) \in U_{\rho}\right\}$. Conversely, let $\eta \in \bigcap_{\rho=1}^{\infty} \liminf _{m \rightarrow \infty}\left\{\eta \in C_{0}: \Psi_{m}(\eta) \in U_{\rho}\right\}$. Then for every $\rho \geq 1$, there is an $\bar{m}_{\rho} \geq 1$ such that $\Psi_{m}(\eta) \in \stackrel{\rho}{U}_{\rho}$ for all $m \geq \bar{m}$. Taking $m \rightarrow \infty$ gives $\eta(\bar{\theta}, \bar{x}) \in U_{\rho}$ for all $\rho \geq 1$, i.e., $\eta(\bar{\theta}, \bar{x}) \in \bigcap_{\rho=1}^{\infty} U_{\rho}$. But $I^{c}=\bigcap_{\rho=1}^{\infty} U_{\rho}$, so $\eta(\bar{\theta}, \bar{x}) \in I^{c}$. This proves our claim.

Since the sets $\left\{\eta \in C_{0}: \Psi_{m}(\eta) \in U_{\rho}\right\}$ are clearly in $\sigma(\mathbb{Q} \mathbb{T})$, it follows that $\left\{\eta \in C_{0}: \eta(\bar{\theta}, \bar{x}) \in\right.$ $\left.I^{c}\right\} \in \sigma(\mathbb{Q} \mathbb{T})$. Therefore $\sigma(\mathbb{Q} \mathbb{T})=\mathscr{B}\left(C_{0}\right)$.

\section{The Weak Infinitesimal Generator $A$}

Let $\left(P^{t}, t \geq 0\right), P^{t}: \mathfrak{e}_{b} \rightarrow \mathfrak{e}_{b}$, be the semigroup associated with the Markov solution process $\left(T^{t}, t \geq 0\right)$ defined by $P^{t}(\Psi)(\eta)=E\left[\Psi\left(T^{t}(\eta)\right)\right], \Psi \in \mathcal{C}_{b}, \eta \in C_{0}$ and $t \geq 0$. where

In this section, we investigate the weak infinitesimal generator $\mathcal{A}$ of the semigroup $\left(P^{t}, t \geq 0\right)$,

$$
\mathcal{A}(\Psi)=w-\lim _{t \rightarrow 0+} \frac{P^{t}(\Psi)-\Psi}{t}
$$

Let $D(\mathcal{A})$ be the set of all $\Psi \in \mathrm{e}_{b}$ for which the above weak limit exists. Note that $w$ $\lim _{t \rightarrow 0+} P^{t}(\Psi)=\Psi, \Psi \in \mathrm{C}_{b}$, by the continuity of the sample paths and dominated convergence theorem.

The following lemmas are needed.

Lemma 4.1: There is a constant $K>0$ (independent of $t, \eta$ ) such that $\left\|\frac{1}{t} E\left(T_{\sim}^{t}(\eta)-\bar{\eta}_{t}\right)\right\| \leq$ $K$ for all $t>0$ and $\eta \in C_{0}$. Also $\lim _{t \rightarrow 0+0} \frac{1}{t} E\left[T^{t}(\eta)-\bar{\eta}_{t}\right]=\tilde{f}(\eta) \otimes \iota, \eta \in C_{0}$, where $\tilde{f}: C_{0} \rightarrow C(L)$ is defined by $\tilde{f}(\eta)(x)=f(x, \eta(\cdot, x))$ and $\tilde{f}(\eta) \otimes \iota \in C(J \times L)$ is defined by $\tilde{f}(\eta) \otimes \iota(\theta, x)=$ $\widetilde{f}(\eta)(x) \iota(\theta)$.

Proof: Let $K>0$ be such that $|f(x, \phi)| \leq K$ for all $x \in L$ and $\phi \in C(J)$. Now, if $t+\theta<0$ and $t>0$, then $T^{t}(\eta)(\theta, x)-\bar{\eta}_{t}(\theta, x)=0$. If $\theta=0$ and $t>0$ then 


$$
\begin{gathered}
T^{t}(\eta)(0, x)-\bar{\eta}_{t}(0, x)=\int_{0}^{t} \int_{0}^{\ell} G(t-s, x, y) f\left(y, T^{s}(\eta)(\cdot, y)\right) d y d s \\
+\int_{0}^{t} \int_{0}^{\ell} G(t-s, x, y) g\left(y, T^{s}(\eta)(\cdot, y)\right) W(d y, d s) .
\end{gathered}
$$

Note that $E\left[\frac{1}{t}\left(T^{t}(\eta)(0, x)-\bar{\eta}_{t}(0, x)\right)\right]$

$$
=E\left[\frac{1}{t} \int_{0}^{t} \int_{0}^{\ell} G(t-s, x, y) f\left(y, T^{s}(\eta)(\cdot, y)\right) d y d s\right]
$$

using the martingale property of the Ito integral. Therefore,

$$
\begin{gathered}
\left|\frac{1}{t} E\left[T^{t}(\eta)(\theta, x)-\bar{\eta}_{t}(\theta, x)\right]\right| \leq \frac{1}{t} E \int_{0}^{t} \int_{0}^{\ell}\left[G(t-s, x, y)\left|f\left(y, T^{s}(\eta)(\cdot, y)\right)\right|\right] d y d s \\
\leq K \frac{1}{t} \int_{0}^{t} \int_{0}^{\ell} G(t-s, x, y) d y d s \\
=K, \text { for all } t>0,(\theta, x) \in J \times L \text { and } \eta \times C_{0} .
\end{gathered}
$$

This shows that $\left\|\frac{1}{t} E\left(T^{t}(\eta)-\bar{\eta}_{t}\right)\right\| \leq K$ for all $t>0$ and $\eta \in C_{0}$. Consequently, for $-r \leq \theta<0$, we have $\lim _{t \rightarrow 0+} \frac{1}{t} E\left[T^{t}(\eta)(\theta, x)-\bar{\eta}_{t}(\theta, \bar{x})\right]=0$ and for $\theta=0$, we have

$$
\begin{gathered}
\lim _{t \rightarrow 0+} E\left[\frac{1}{t}\left(T^{t}(\eta)(\theta, x)-\bar{\eta}_{t}(\theta, x)\right)\right] \\
=\lim _{t \rightarrow 0+} \frac{1}{t} \int_{0}^{t} \int_{0}^{\ell} G(t-s, x, y) E\left[F\left(y, T^{s}(\eta)(\cdot, y)\right)\right] d y d s \\
=\lim _{t \rightarrow 0+} \frac{1}{t} \int_{0}^{t} \int_{0}^{\ell} \sum_{n=1}^{\infty} e^{-\lambda_{n}(t-s)} \phi_{n}(x) \phi_{n}(y) E\left[f\left(y, T^{s}(\eta)(\cdot, y)\right)\right] d y d s \\
+\lim _{t \rightarrow 0+} \int_{0}^{t} \int_{0}^{\ell} \sum_{n=1}^{\ell}\left(-\lambda_{n}\right) e^{-\lambda_{n}(t-s)} \phi_{n}(x) \phi_{n}(y) E\left[f\left(y, T^{s}(\eta)(\cdot, y)\right)\right] d y d s \\
=\int_{0}^{\ell} \sum_{n=1}^{\infty} \phi_{n}(x) \phi_{n}(y) f(y, \eta(\cdot, y)) d y=\tilde{f}(\eta)(x) .
\end{gathered}
$$

This shows that for $\alpha \in C_{0}^{*}, \quad \lim _{t \rightarrow 0+} \frac{1}{t} E\left[\alpha\left(T^{t}(\eta)-\bar{\eta}_{t}\right)\right]=\bar{\alpha}(\tilde{f}(\eta) \otimes \iota)$ where $\bar{\alpha}: C_{0} \rightarrow C_{0} \otimes \Lambda$ is the extension of $\alpha \in C_{0}^{*}$ as defined in Lemma 2.2 . 
Lemma 4.2: Let $\beta$ be a continuous bilinear form on $C_{0}$. Then

$$
\begin{gathered}
\lim _{t \rightarrow 0+}\left[\frac{1}{t} E \beta\left(T^{t}(\eta)-\bar{\eta}_{t}, T^{t}(\eta)-\bar{\eta}_{t}\right)\right. \\
-\frac{1}{t} E \beta\left(\int_{0}^{t+\cdot} \int_{0}^{\ell} G(t+\cdot-s, *, y) g(y, \eta(\cdot, y)) W(d y, d s),\right. \\
\left.\left.\int_{0}^{t+\cdot} \int_{0}^{\ell} G(t+\cdot-s, *, y) g(y, \eta(\cdot, y)) W(d y, d s)\right)\right]=0 .
\end{gathered}
$$

Proof: We prove first that

$$
\lim _{t \rightarrow 0+} E\left\|\frac{1}{\sqrt{t}}\left(T^{t}(\eta)-\bar{\eta}_{t}\right)-\frac{1}{\sqrt{t}} \gamma(g, \eta)(t)\right\|^{2}=0,
$$

where

$$
\gamma(g, \eta)(t)(\theta, x)=\left\{\begin{array}{c}
\int_{0}^{t+\theta} \int_{0}^{\ell} G(t+\theta-s, x, y) g(y, \eta(\cdot, y)) W(d y, d s), \text { if }(\theta, x) \in[-t, 0] \times L, \\
0 \text { if }(\theta, x) \in[-r,-t) \times L .
\end{array}\right.
$$

Observe that for $0<t<r$, and $(\theta, x) \in[-t, 0] \times L$,

$$
\begin{aligned}
& \frac{1}{\sqrt{t}}\left(T^{t}(\eta)(\theta, x)-\bar{\eta}_{t}(\theta, x)\right)-\frac{1}{\sqrt{t}} \gamma(g, \eta)(t)(\theta, x) \\
= & \frac{1}{\sqrt{t}} \int_{0}^{t+\theta} \int_{0}^{\ell} G(t+\theta-s, x, y) f\left(y, T^{s}(\eta)\right)(\cdot, y) d y d s \\
+ & \frac{1}{\sqrt{t}} \int_{0}^{t+\theta} \int_{0}^{\ell} G(t+\theta-s, x, y) g\left(y, T^{s}(\eta)\right)(\cdot, y) W(d y, d s) \\
- & \frac{1}{\sqrt{t}} \int_{0}^{t+\theta} \int_{0}^{\ell} G(t+\theta-s, x, y) g(y, \eta(\cdot, y)) W(d y, d s) .
\end{aligned}
$$

Therefore,

$$
\begin{gathered}
E\left\|\frac{1}{\sqrt{t}}\left(T^{t}(\eta)-\bar{\eta}_{t}\right)-\frac{1}{\sqrt{t}} \gamma(g, \eta)(t)\right\|^{2} \\
\leq 2 \frac{1}{t} E\left\{\sup _{(\theta, x) \in J \times L}\left|\int_{0}^{t+\theta} \int_{0}^{\ell} G(t+\theta-s, x, y) f\left(y, T^{s}(\eta)(\cdot, y)\right) d y d s\right|^{2}\right\} \\
+2 \frac{1}{t} E\left\{\sup _{(\theta, x) \in J \times L}\left|\int_{0}^{t+\theta} \int_{0}^{\ell} G(t+\theta-s, x, y)\left[g\left(y, T^{s}(\eta)(\cdot, y)\right)-g(y, \eta(\cdot, y))\right] W(d y, d s)\right|^{2}\right\}
\end{gathered}
$$




$$
\begin{aligned}
& \leq 2 \frac{1}{t} E\left\{\sup _{(\theta, x) \in J \times L}\left(\int_{0}^{t+\theta} \int_{0}^{\ell} G^{2}(t+\theta-s, x, y) d y d s\right)\left(\int_{0}^{t+\theta} \int_{0}^{\ell} f^{2}\left(y, T^{s}(\eta)(\cdot, y)\right) d y d s\right)\right\} \\
& +2 \frac{1}{t} \int_{0}^{t} \int_{0}^{\ell} \sup _{(\theta, x) \in J \times L} G^{2}(t+\theta-s, x, y)\left[g\left(y, T^{s}(\eta)(\cdot, y)\right)-g(y, \eta(\cdot, y))\right]^{2} d y d s \\
& \leq 2 \frac{K^{2}}{t \ell} \sum_{k=1}^{\infty} \frac{1}{\lambda_{k}}\left(1-e^{-2 \lambda k^{t}}\right)+8 \frac{K^{2}}{t \ell} \sum_{k=1}^{\infty} \frac{1}{\lambda_{k}}\left(1-e^{-2 \lambda_{k} t}\right) \\
& =\frac{10 K^{2}}{\ell t} \sum_{k=1}^{\infty} \frac{1}{\lambda_{k}}\left(1-e^{-2 \lambda_{k} t}\right) \rightarrow 0 \text { as } t \rightarrow 0+, \\
& \gamma(g, \eta)(t)(\theta, x)=\int_{0}^{t+\theta} \int_{0}^{\ell} G(t+\theta-s, x, y) g(y, \eta(\cdot, y)) W(d y, d s) \\
& =\int_{0}^{t+\theta} \int_{0}^{\ell} \sum_{n=1}^{\infty} \exp \left(-\lambda_{n}(t+\theta-s)\right) \phi_{n}(x) \phi_{n}(y) g(y, \eta(\cdot, y)) W(d y, d s) \\
& \left.=\sum_{n=1}^{\infty} \phi_{n}(x) \int_{0}^{t+\theta} \int_{0}^{\ell} \exp \left(-\lambda_{n} t+\theta-s\right)\right) \phi_{n}(y) g(y, \eta(\cdot, y)) W(d y, d s) \\
& =\sum_{n=1}^{\infty} \phi_{n}(x) \int_{0}^{t+\theta} \exp \left(-\lambda_{n}(t+\theta-s)\right) d B_{n}(d s), \text { if }(\theta, x) \in[-t, 0] \times L .
\end{aligned}
$$

This shows that $\gamma(g, \eta)(\theta, x)=\chi([-t, 0] \times L) \sum_{n=1}^{\infty} \phi_{n}(x) \int_{0}^{t+\theta} \exp \left(-\lambda_{n}(t+\theta-s)\right) d B_{n}(s)$, where $B_{n}(t)=\int_{0}^{t} \phi_{n}(y) g(y, \eta(\cdot, y)) W(d y, d s)$ is a Brownian motion with mean zero and $E\left[B_{n}^{2}(t)\right]=\sigma_{n}^{2} t$, with $\sigma_{n}^{2}:=\int_{0}^{\ell} \phi_{n}^{2}(y) g^{2}(y, \eta(\cdot, y)) d y$.

Now, since $\beta$ is bilinear,

$$
\begin{gathered}
\frac{1}{t} \beta\left\langle T^{t}(\eta)-\bar{\eta}_{t}, T^{t}(\eta)-\bar{\eta}_{t}\right\rangle-\frac{1}{t} \beta\langle\gamma(g, \eta)(t), \gamma(g, \eta)(t)\rangle \\
=\beta\left\langle\frac{1}{\sqrt{t}}\left(T^{t}(\eta)-\bar{\eta}_{t}\right)-\frac{1}{\sqrt{t}} \gamma(g, \eta)(t), \frac{1}{\sqrt{t}}\left(T^{t}(\eta)-\bar{\eta}_{t}\right)-\frac{1}{\sqrt{t}} \gamma(g, \eta)(t)\right\rangle \\
+\beta\left\langle\frac{1}{\sqrt{t}}\left(T^{t}(\eta)-\bar{\eta}_{t}-\gamma(g, \eta)(t)\right), \frac{1}{\sqrt{t}} \gamma(g, \eta)(t)\right\rangle \\
+\beta\left\langle\frac{1}{\sqrt{t}} \gamma(g, \eta)(t), \frac{1}{\sqrt{t}}\left(T^{t}(\eta)-\bar{\eta}_{t}-\gamma(g, \eta)(t)\right)\right\rangle .
\end{gathered}
$$

Thus, by continuity of $\beta$ and Hölder inequality, one obtains: 


$$
\begin{gathered}
\left|\frac{1}{t} E \beta\left\langle T^{t}(\eta)-\bar{\eta}_{t}, T^{t}(\eta)-\bar{\eta}_{t}\right\rangle-\frac{1}{t} E \beta\langle\gamma(g, \eta)(t), \gamma(g, \eta)(t)\rangle\right| \\
\leq\|\beta\| E\left\|\frac{1}{\sqrt{t}}\left(T^{t}(\eta)-\bar{\eta}_{t}-\gamma(g, \eta)(t)\right)\right\|^{2} \\
+2\|\beta\|\left[E\left\|\frac{1}{\sqrt{t}}\left(T^{t}(\eta)-\bar{\eta}_{t}-\gamma(g, \eta)(t)\right)\right\|^{2}\right]^{1 / 2} \cdot\left[E\left\|\frac{1}{\sqrt{t}} \gamma(g, \eta)(t)\right\|^{2}\right]^{1 / 2}
\end{gathered}
$$

for all $t>0$. But $\left\|\frac{1}{\sqrt{t}} \gamma(g, \eta)(t)\right\|^{2}$

$$
\begin{gathered}
=\frac{1}{t} E\left\{\sup _{(\theta, x) \in J \times L}|\gamma(g, \eta)(t)(\theta, x)|^{2}\right\} \\
\leq \frac{1}{t} \quad \sup _{(\theta, x) \in J \times L} \int_{0}^{t+\theta} \int_{0}^{\ell} G^{2}(t+\theta-s, x, y) g^{2}(y, \eta(\cdot, y)) d y d s \\
\leq \frac{K^{2}}{t} \sup _{(\theta, x) \in J \times L} \int_{0}^{t+\theta} \int_{0}^{\ell} G^{2}(t+\theta-s, x, y) d y d s \\
\leq \frac{K^{2}}{\ell t} \sum_{k=1}^{\infty} \frac{1}{\lambda_{k}}\left(1-e^{-2 \lambda_{k} t}\right)<\infty \text { for all } t>0 .
\end{gathered}
$$

This shows that

$$
\lim _{t \rightarrow 0+}\left[\frac{1}{t} E \beta\left\langle T^{t}(\eta)-\bar{\eta}_{t}, T^{t}(\eta)-\bar{\eta}_{t}\right\rangle-\frac{1}{t} E \beta\langle\gamma(g, \eta)(t), \gamma(g, \eta)(t)\rangle\right]=0 .
$$

Lemma 4.3: For any continuous bilinear form $\beta$ on $C_{0}$,

$$
\lim _{t \rightarrow 0+} \frac{1}{t} E \beta\left\langle T^{t}(\eta)-\bar{\eta}_{t}, T^{t}(\eta)-\bar{\eta}_{t}\right\rangle=\sum_{n=1}^{\infty} \beta\left\langle\sigma_{n} \phi_{n}, \sigma_{n} \phi_{n}\right\rangle
$$

where $\sigma_{n}^{2}=\int_{0}^{\ell} \phi_{n}^{2}(y) g^{2}(y, \eta(\cdot, y)) d y$.

Proof: In view of the preceding lemma, it suffices to show that

$$
\lim _{t \rightarrow 0+} \frac{1}{t} E \beta\langle\gamma(g, \eta)(t), \gamma(g, \eta)(t)\rangle=\sum_{n=1}^{\infty} \beta\left\langle\sigma_{n} \phi_{n}, \sigma_{n} \phi_{n}\right\rangle .
$$

If $\xi, \eta \in C_{0}$, let $\xi \otimes \eta$ be the function from $J \times L \times J \times L \rightarrow \mathbb{R}$ defined by

$$
(\xi \otimes \eta)(\theta, x, \tau, y)=\xi(\theta, x) \eta(\tau, y) \text { for all }(\theta, x, \tau, y) \in J \times L \times J \times L .
$$
The projective tensor product $C_{0} \otimes{ }_{\pi} C_{0}$ is the vector space of all functions of the form $\sum_{i=1}^{N} \xi_{i} \otimes \eta_{i}$
where $\xi_{i}, \eta_{i} \in C_{0}, i=1,2, \ldots, N$. It carries the norm

$$
\|h\|_{\otimes_{\pi}}=\inf \left\{\sum_{i=1}^{N}\left\|\xi_{i}\right\|\left\|\eta_{i}\right\|: h=\sum_{i=1}^{N} \xi_{i} \otimes \eta_{i}, \xi_{i}, \eta_{i} \in C_{0}, i=1,2, \ldots, N\right\}
$$

where the infimum is taken over all possible finite representations of $h \in C_{0} \otimes{ }_{\pi} C_{0}$. Denote by $C_{0} \widehat{\otimes}_{\pi} C_{0}$ the completion of $C_{0} \otimes{ }_{\pi} C_{0}$ under the above norm. It is well known from [15] that $C_{0} \widehat{\otimes}_{\pi} C_{0}$ is continuous and densely embedded in $C_{0}(J \times L \times J \times L, \mathbb{R})$, the Banach space (under the sup-norm) of all continuous functions $\Gamma: J \times L \times J \times L \rightarrow \mathbb{R}$ with $\Gamma(\theta, 0, \tau, y)=\Gamma(\theta, \ell, \tau, y)=$ 
$\Gamma(\theta, x, \tau, 0)=\Gamma(\theta, x, \tau, \ell)=0$ for all $x, y \in L$.

Since $C_{0}$ is a separable Banach space, so is $C_{0} \widehat{\otimes}_{\pi} C_{0}$. Let $\Psi \subset C_{0}$ be a countable dense subset of $C_{0}$, then the countable set

$$
\Psi \otimes \Psi=\left\{\sum_{i=1}^{N} \xi_{i} \otimes \eta_{i}: \xi_{i}, \eta_{i} \in \Psi, i=1, \ldots, N, N=1,2, \ldots\right\}
$$

is dense in $C_{0} \otimes{ }_{\pi} C_{0}$ and hence in $C_{0} \widehat{\otimes}_{\pi} C_{0}$.

The continuous bilinear form $\beta$ on $C_{0}$ corresponds to a continuous linear functional $\tilde{\beta} \in$ $\left(C_{0} \widehat{\otimes}_{\pi} C_{0}\right)^{*}$.

Now let $\psi_{1}, \psi_{2} \in \mathcal{L}^{2}\left(\Omega, C_{0}\right)$, where $\mathcal{L}^{2}\left(\Omega, C_{0}\right)$ is the space of $C_{0}$-valued random variables $\Theta$ defined on $(\Omega, \Im, \Omega)$ such that $\int_{\Omega}\|\Theta(\omega)\|^{2} d P<\infty$. The map $C_{0} \otimes C_{0} \rightarrow C_{0} \otimes{ }_{\pi} C_{0},(\xi, \eta) \mapsto \xi \otimes \eta$ is clearly continuous bilinear.

Thus $\psi_{1}(\cdot) \otimes \psi_{2}(\cdot): \Omega \rightarrow C_{0} \widehat{\otimes}_{\pi} C_{0}, \omega \mapsto \psi_{1}(\omega) \otimes \psi_{2}(\omega)$ is Borel measurable. But $\| \psi_{1}(\omega) \otimes$ $\psi_{2}(\omega)\left\|_{\otimes} \leq\right\| \psi_{1}(\omega)\|\| \psi_{2}(\omega) \|$ for almost all $\omega \in \Omega$; hence by Hölder inequality, the integral $\int_{\Omega}\left\|\psi_{1}(\omega) \otimes \psi_{2}(\omega)\right\|_{\otimes_{\pi}} d P(\omega)$ exists and

$$
\int_{\Omega}\left\|\psi_{1}(\omega) \otimes \psi_{2}(\omega)\right\|_{\otimes_{\pi}} d P(\omega) \leq\left[\int_{\Omega}\left\|\psi_{1}(\omega)\right\|^{2} d P(\omega)\right]^{1 / 2}\left[\int_{\Omega}\left\|\psi_{2}(\omega)\right\|^{2} d P(\omega)\right]^{1 / 2}
$$

From the separability of $C_{0} \otimes{ }_{\pi} C_{0}$, the Bochner integral

$$
E\left[\psi_{1}(\cdot) \otimes \psi_{2}(\cdot)\right]=\int_{\Omega} \psi_{1}(\omega) \otimes \psi_{2}(\omega) d P(\omega)
$$

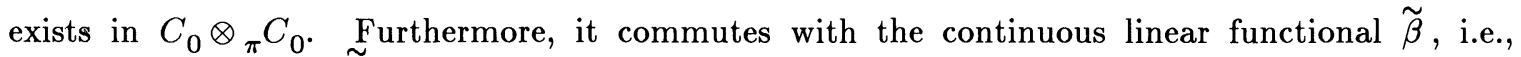
$E \beta\left\langle\psi_{1}(\cdot), \psi_{2}(\cdot)\right\rangle=E \tilde{\beta}\left(\psi_{1}(\cdot) \otimes \psi_{2}(\cdot)\right)$.

For $0<t<r$ and $(\theta, x),(\tau, y) \in J \times L$,

$$
\begin{gathered}
E[\gamma(g, \eta)(t) \otimes \gamma(g, \eta)(t)((\theta, x),(\tau, y))]=E[\gamma(g, \eta)(t)(\theta, x) \gamma(g, \eta)(t)(\tau, y))] \\
=E\left[\int_{0}^{t+\theta} \int_{0}^{\ell} G(t+\theta-s, x, z) g(z, \eta(\cdot, z)) d W(d z, d s)\right. \\
\left.\cdot \int_{0}^{t+\tau} \int_{0}^{\ell} G(t+\tau-s, y, z) g(z, \eta(\cdot, z)) d W(d z, d s)\right] \\
=E\left[\sum_{j=1}^{\infty} \phi_{j}(x) \int_{0}^{t+\theta} \exp \left(-\lambda_{j}(t+\theta-s)\right) d B_{j}(s) \cdot \sum_{k=1}^{\infty} \phi_{k}(y) \int_{0}^{t+\tau} \exp \left(\left(-\lambda_{k}(t+\tau-s) d B_{k}(s)\right)\right]\right. \\
=\sum_{n=1}^{\infty} \phi_{n}(x) \phi_{n}(y) \int_{0}^{t+\min (\theta, \tau)} \exp \left(-\lambda_{n}(2 t+\theta+\tau-2 s)\right) \sigma_{n}^{2} d s \\
=\frac{1}{2} \sum_{k=1}^{\infty} \frac{\sigma_{n}^{2}}{\lambda_{n}}\left[e^{-\lambda_{n}[\max (\theta, \tau)-\min (\theta, \tau)]}-e^{\left.-\lambda_{n}(2 t+\theta+\tau)\right] \phi_{n}(x) \phi_{n}(y) .}\right.
\end{gathered}
$$


Let $(*) K(\theta, x ; \tau, y)=\frac{1}{t} E[\gamma(g, \eta)(t) \otimes \gamma(g, \eta)(t)(\theta, x ; \tau, y)]$

$$
=\frac{1}{2} \sum_{n=1}^{\infty} \frac{\sigma_{n}^{2}}{\lambda_{n} t}\left[e^{-\lambda_{n}(\max (\theta, \tau)-\min (\theta, \tau)}-e^{-\lambda_{n}(2 t+\theta+\tau)}\right] \cdot \phi_{n}(x) \phi_{n}(y) \chi_{[-t, 0]}(\theta) \chi_{[-t, 0]}(\tau) .
$$

Since $\gamma(g, \eta)(t) \in \mathcal{L}^{2}\left(\Omega, C_{0}\right)$, is clear from $(*)$ that $K \in C_{0} \widehat{\otimes}_{\pi} C_{0}$ and

$$
E \beta\left\langle\frac{1}{\sqrt{t}} \gamma(g, \eta)(t), \frac{1}{\sqrt{t}} \gamma(g, \eta)(t)\right\rangle=\tilde{\beta}(K) .
$$

A simple calculation using $(*)$ and the fact that $\tilde{\beta} \in\left(C_{0} \widehat{\otimes}_{\pi} C_{0}\right)^{*}$ yields

$$
\begin{gathered}
\lim _{t \rightarrow 0+} \tilde{\beta}(K)=\sum_{n=1}^{\infty} \sigma_{n}^{2} \bar{\beta}\left\langle\phi_{n} \otimes \iota, \phi_{n} \otimes \iota\right\rangle \\
=\frac{1}{2} \sum_{n=1}^{\infty} \phi_{n}(x) \phi_{n}\left(x^{\prime}\right) \sigma_{n}^{2} \frac{1}{\lambda_{n}} \cdot\left(1-e^{-2 \lambda_{n} t}\right),
\end{gathered}
$$

i.e. $K_{t}=E\langle\gamma(g, \eta)(t) \otimes \gamma(g, \eta)(t)\rangle$.

Since $\gamma(g, \eta)(t) \in \mathcal{L}^{2}\left(\Omega, C_{0}\right)$, it is clear from $(*)$ that

$$
E \beta\langle\gamma(g, \eta)(t), \gamma(g, \eta)(t)\rangle=\bar{\beta}\left(K_{t}\right) .
$$

Therefore, $\quad \lim _{t \rightarrow 0+} \frac{1}{t} E \beta\langle\gamma(g, \eta)(t), \gamma(g, \eta)(t)\rangle$

$$
=\bar{\beta}\left(\sum_{n=1}^{\infty} \phi_{n}(x) \phi_{n}\left(x^{\prime}\right) \sigma_{n}^{2}\right)=\sum_{n=1}^{\infty} \bar{\beta}\left\langle\sigma_{n} \phi_{n}, \sigma_{n} \phi_{n}\right\rangle,
$$

where $\sigma_{n}^{2}=\int_{0}^{\ell} \phi_{n}^{2}(y) g^{2}(y, \eta(\cdot, y)) d y$. This proves the lemma.

Let $D(\varphi) \subset C_{b}^{0}$ be the domain for the infinitesimal generator $\varphi$ of the semigroup $\left(S^{t}, t \geq 0\right)$, where $\varphi: D(\varphi) \rightarrow \mathrm{C}_{b}$ is defined by

$$
\varphi(\Psi)(\eta)=\lim _{t \rightarrow 0+} \frac{1}{t}\left[S^{t}(\Psi)(\eta)-\Psi(\eta)\right] .
$$

In order to study the weak infinitesimal generator $\mathcal{A}$ of the semigroup of Markov operators $\left(P^{t}, t \geq 0\right)$ for the solution maps $\left(T^{t}, t \geq 0\right)$ of equation (1.1), we need the following conditions:

$(C 1) \quad \Psi \in D(\varphi)$

(C2) $\Psi$ is $C^{2}$ (twice Frechet differentiable);

(C3) The Frechet derivatives $D \Psi$ and $D^{2} \Psi$ are globally bounded;

(C4) $D^{2} \Psi$ is globally Lipschitz on $C_{0}$.

Theorem 4.1: Suppose $\Psi: C_{0} \rightarrow \mathbb{R}$ satisfies conditions $(C 1)-(C 4)$. Then $\Psi \in D(\mathcal{A})$ and for each $\eta \in C_{0}$,

$$
\mathcal{A}(\Psi)(\eta)=\varphi(\Psi)(\eta)+D \Psi(\eta)(\tilde{f}(\eta) \otimes \iota)+\frac{1}{2} \sum_{n=1}^{\infty} \sigma_{n}^{2} \overline{D^{2} \Psi(\eta)}\left\langle\phi_{n} \otimes \iota, \phi_{n} \otimes \iota\right\rangle,
$$

where

$$
\sigma_{n}^{2}=\int_{0}^{\ell} \phi_{n}^{2}(y) g^{2}(y, \eta(\cdot, y)) d y .
$$

Proof: Fix $\eta \in C_{0}$ and let $\left(T^{t}(\eta), t \geq 0\right)$ be the solution process of equation (1.1) through the initial datum $\eta$. Suppose $\Psi \in D(\mathcal{A})$. 
Since $\Psi$ is $C^{2}$, then by Taylor's theorem (see e.g. [8]),

$$
\Psi\left(T^{t}(\eta)\right)-\Psi(\eta)=\Psi\left(\tilde{\eta}_{t}\right)-\Psi(\eta)+D \Psi\left(\bar{\eta}_{t}\right)\left(T^{t}(\eta)-\bar{\eta}_{t}+R_{2}(t)\right), \text { a.s. } t>0
$$

where

$$
R_{2}(t)=\int_{0}^{1}(1-\lambda) D^{2} \Psi\left(\bar{\eta}_{t}+\lambda\left(T^{t}(\eta)-\bar{\eta}_{t}\right)\right)\left\langle T^{t}(\eta)-\bar{\eta}_{t}, T^{t}(\eta)-\bar{\eta}_{t}\right\rangle d \lambda \text { a.s. }
$$

Taking expectations, we obtain

$$
\frac{1}{t} E\left[\Psi\left(T^{t}(\eta)\right)-\Psi(\eta)\right]=\frac{1}{t}\left[S^{t}(\Psi)(\eta)-\Psi(\eta)\right]+\frac{1}{t} E D \Psi\left(\bar{\eta}_{t}\right)\left(T^{t}(\eta)-\bar{\eta}_{t}\right)+\frac{1}{t} E R_{2}(t) .
$$

Note that $\lim _{t \rightarrow 0+} \frac{1}{t}\left[S^{t}(\Psi)(\eta)-\Psi(\eta)\right]=\varphi(\Psi)(\eta)$.

We need to evaluation the following two limits

$$
\lim _{t \rightarrow 0+} \frac{1}{t} E\left(D \Psi\left(\bar{\eta}_{t}\right)\left(T^{t}(\eta)-\bar{\eta}_{t}\right)\right.
$$

and

$$
\lim _{t \rightarrow 0+} \frac{1}{t} E R_{2}(t)
$$

We first evaluate (4.1). From the proof of Lemma 4.1, there exists a $K>0$ such that

$$
\left\|\frac{1}{t} E\left(T^{t}(\eta)-\bar{\eta}_{t}\right)\right\| \leq K \text { for all } t>0 \text { and all } \eta \in C_{0} .
$$

Hence,

$$
\begin{aligned}
& \mid \frac{1}{t}\left(E\left[\Psi\left(\bar{\eta}_{t}\right)\left(T^{t}(\eta)-\bar{\eta}_{t}\right)\right]\right)-\frac{1}{t} E\left[\left(D \Psi(\eta)\left(T^{t}(\eta)-\bar{\eta}_{t}\right)\right] \mid\right. \\
& =\mid D \Psi\left(\bar{\eta}_{t}\right)\left[\frac{1}{t} E\left(T^{t}(\eta)-\bar{\eta}_{t}\right)\right]-D \Psi(\eta)\left[\frac{1}{t} E\left[T^{t}(\eta)-\eta_{t}\right] \mid\right. \\
& \leq K\left\|D \Psi\left(\bar{\eta}_{t}\right)-D \Psi(\eta)\right\| \text { for all } t>0 .
\end{aligned}
$$

Letting $t \rightarrow 0+$ and using the continuity of $D \Psi$ at $\eta$, we obtain

$$
\begin{aligned}
& \lim _{t \rightarrow 0+} \frac{1}{t} E\left[D \Psi\left(\bar{\eta}_{t}\right)\left(T^{t}(\eta)-\bar{\eta}_{t}\right)\right] \\
= & \lim _{t \rightarrow 0+} \frac{1}{t} D \Psi(\eta) E\left[T^{t}(\eta)-\bar{\eta}_{t}\right] \\
= & D \Psi(\eta) \tilde{f}(\eta) \otimes \iota) \text { by Lemma 4.1. }
\end{aligned}
$$

Second, we look at the limit (4.2). Observe that if $K$ is a bound for $f, g: L \times C(J) \rightarrow \mathbb{R}$ and if $t>0$ is small, then

$$
\begin{aligned}
& E\left\|T^{t}(\eta)-\bar{\eta}_{t}\right\|^{4}=E \sup _{(\theta, x) \in J \times L} \mid \int_{0}^{t+\theta} \int_{0}^{\ell} G(t-s+\theta, x, y) f\left(y, T^{s}(\eta)(\cdot, y)\right) d y d s \\
& -\left.\int_{0}^{t+\theta} \int_{0}^{\ell} G(t+\theta-s, x, y) g\left(y, T^{s}(\eta)(\cdot, y)\right) W(d y, d s)\right|^{4} \\
& \leq 8 E \sup _{(\theta, x) \in J \times L}\left|\int_{0}^{t+\theta} \int_{0}^{\ell} G(t+\theta-s, x, y) f\left(y, T^{s}(\eta)(\cdot, y)\right) d y d s\right|^{4}
\end{aligned}
$$




$$
\begin{gathered}
+8 \underset{(\theta, x) \in J \times L}{E} \sup _{0}\left|\int_{0}^{t+\theta} \int_{0}^{\ell} G(t+\theta-s, x, y) g\left(y, T^{s}(\eta)(\cdot, y)\right) W(d y, d s)\right|^{4} \\
\leq 8 K^{2} t^{4}+8 K_{2} t \int_{0}^{t} \int_{0}^{\ell} E\left\|G(t-s, x, y) g\left(y, T^{s}(\eta)(y)\right)\right\|^{4} d y d s \\
\quad \leq 8 K^{2} t^{4}+8 K_{2} K^{4} t \int_{0}^{t} \int_{0}^{\ell} G^{4}(t-s, x, y) d y d s:=\Delta(t)
\end{gathered}
$$

where we have used the property of the Itô integral (see e.g. [16]). Note furthermore that, if $\lambda \in[0,1]$,

$$
\begin{gathered}
\mid \frac{1}{t} E D^{2} \Psi\left(\bar{\eta}_{t}+\lambda\left(T^{t}(\eta)-\bar{\eta}_{t}\right)\right)\left\langle T^{t}(\eta)-\bar{\eta}_{t}, T^{t}(\eta)-\bar{\eta}_{t}\right\rangle \\
-\frac{1}{t} E D^{2} \Psi(\eta)\left\langle T^{t}(\eta)-\bar{\eta}_{t}, T^{t}(\eta)-\bar{\eta}_{t}\right\rangle \\
\leq \frac{1}{t} E\left\{\left\|D^{2} \Psi\left(\bar{\eta}_{t}+\lambda\left(T^{t}(\eta)-\bar{\eta}_{t}\right)\right)-D^{2} \Psi(\eta)\right\|\left\|T^{t}(\eta)-\bar{\eta}_{t}\right\|^{2}\right\} \\
\leq\left\{E\left[\left\|D^{2} \Psi\left(\bar{\eta}+\lambda\left(T^{t}(\eta)-\bar{\eta}\right)\right)-D^{2} \Psi(\eta)\right\|^{2}\right]\right\}^{1 / 2} \cdot\left\{\frac{1}{t^{2}} E\left[\left\|T^{t}(\eta)-\bar{\eta}_{t}\right\|^{4}\right]\right\}^{1 / 2} \\
\leq\left\{\frac{1}{t^{2}} \Delta(t)\right\}^{1 / 2}\left\{E\left[\left\|D^{2} \Psi\left(\bar{\eta}_{t}+\lambda\left(T^{t}(\eta)-\bar{\eta}_{t}\right)\right)-D^{2} \Psi(\eta)\right\|^{2}\right\}^{1 / 2} .\right.
\end{gathered}
$$

But $D^{2} \Psi$ is globally Lipschitz, with Lipschitz constant $k$, so

$$
\begin{gathered}
E\left\|D^{2} \Psi\left(\bar{\eta}_{t}+\lambda\left(T^{t}(\eta)-\bar{\eta}_{t}\right)\right)-D^{2} \Psi(\eta)\right\|^{2} \\
\leq k^{2} E\left(\left\|\bar{\eta}_{t}-\eta\right\|+\left\|T^{t}(\eta)-\bar{\eta}_{t}\right\|\right)^{2} \\
\leq 2 k^{2}\left\|\bar{\eta}_{t}-\eta\right\|^{2}+2 k^{2}\left\{E\left\|T^{t}(\eta)-\bar{\eta}_{t}\right\|^{4}\right\}^{1 / 2} \\
\leq 2 k^{2}\left\|\bar{\eta}_{t}-\eta\right\|^{2}+2 k^{2}(\Delta(t))^{1 / 2}
\end{gathered}
$$

Letting $t \rightarrow 0+$, we have

$$
\begin{gathered}
\lim _{t \rightarrow 0+} \frac{1}{t} E D^{2} \Psi\left(\bar{\eta}_{t}+(1-\lambda)\left(T^{t} \eta-\bar{\eta}_{t}\right)\left\langle T^{t}(\eta)-\bar{\eta}_{t}, T^{t}(\eta)-\bar{\eta}_{t}\right\rangle\right. \\
=\lim _{t \rightarrow 0+} E D^{2} \Psi(\eta) \frac{\left\langle T^{t}(\eta)-\bar{\eta}_{t}, T^{t}(\eta)-\bar{\eta}_{t}\right\rangle}{t}
\end{gathered}
$$

uniformly in $\lambda \in[0,1]$. From this and Lemma 4.3,

$$
\begin{aligned}
& \lim _{t \rightarrow 0+} \frac{1}{t} E R_{2}(t)=\int_{0}^{1}(1-\lambda) \lim _{t \rightarrow 0+} \frac{1}{t} E\left[D^{2} \Psi(\eta)\left\langle T^{t}(\eta)-\bar{\eta}_{t}, T^{t}(\eta)-\bar{\eta}_{t}\right\rangle\right] d \lambda \\
& =\sum_{n=1}^{\infty} \frac{1}{2} \overline{D^{2} \Psi(\eta)}\left\langle\alpha_{n} \phi_{n}, \alpha_{n} \phi_{n}\right\rangle=\frac{1}{2} \sum_{n=1}^{\infty} \sigma_{n}^{2} \overline{D^{2} \Psi(\eta)}\left\langle\phi_{n} \otimes \iota, \phi_{n} \otimes \iota\right\rangle
\end{aligned}
$$

where $\alpha_{n}^{2}=\int_{0}^{\ell} \phi_{n}^{2}(y) g^{2}(y, \eta(\cdot, y)) d y$. 
This proves that

$$
\begin{gathered}
\mathcal{A}(\Psi)(\eta)=\lim _{t \rightarrow 0+} \frac{1}{t}\left(\Psi\left(T^{t}(\eta)\right)-\Psi(\eta)\right) \\
=\varphi(\Psi)(\eta)+D \Psi(\eta)(\tilde{f}(\eta) \otimes \iota)+\frac{1}{2} \sum_{n=1}^{\infty} \sigma_{n}^{2} \overline{D^{2} \Psi(\eta)}\left\langle\phi_{n} \otimes \iota, \phi_{n} \otimes \iota\right\rangle
\end{gathered}
$$

where $\overline{D^{2} \Psi(\eta)}$ is the extension of the bilinear function $D^{2} \Psi(\eta)$ on $C_{0}$ and $\sigma_{n}^{2}=$ $\int_{0}^{\ell} \phi_{n}^{2}(y) g^{2}(y, \eta(y)) d y$.

\section{The Weak Infinitesimal Generator on Quasi-Tame Functions}

In this section, we are interested in the action of the weak infinitesimal generator $\mathcal{A}$ on the class of quasi-tame functions $\mathbb{Q} \mathbb{T}$ as defined in Definition 3.3.

Theorem 5.1: $\mathbb{Q} \mathbb{C} \subseteq D(\mathcal{A})$. If $\Psi \in \mathbb{Q} \mathbb{T}$ is of the form $\Psi(\eta)=h(m(\eta)), \eta \in C_{0}$, where $h: \mathbb{R}^{k} \rightarrow$ $\mathbb{R}, m: C_{0} \rightarrow \mathbb{R}^{k}$,

$$
\begin{gathered}
m(\eta)=\left(\int_{-r}^{0} \int_{0}^{\ell} \alpha_{1}(\eta(\theta, x)) \beta_{1}(\theta, x) d x d \theta, \ldots, \int_{-r}^{0} \int_{0}^{\ell} \alpha_{k-1}(\eta(\theta, x)) \beta_{k-1}(\theta, x) d x d \theta, \int_{0}^{\ell} \eta(0, x) d x\right), \\
\alpha_{i}: \mathbb{R} \rightarrow \mathbb{R} \text { and } \beta_{i}: J \times L \rightarrow \mathbb{R},
\end{gathered}
$$

then

$$
\begin{gathered}
\mathcal{A}(\Psi)(\eta)=\sum_{i=1}^{k-1} D_{i} h(m(\eta))\left[-\int_{-r}^{0} \int_{0}^{\ell} \alpha_{i}(\eta(\theta, x)) \frac{\partial \beta_{i}(\theta, x)}{\partial \theta} d x d \theta\right. \\
\left.+\int_{0}^{\ell} \alpha_{i}\left(\int_{0}^{\ell} \eta(0, y) d y\right) \beta_{i}(0, x) d x-\int_{0}^{\ell} \alpha_{i}(\eta(-r, x)) \beta_{i}(-r, x) d x\right] \\
+D h(m(\eta))\left(\int_{r}^{0} \int_{0}^{\ell} D \alpha_{i}(\eta(\theta, x))(\tilde{f}(\eta) \otimes \iota)(\theta, x) \beta_{1}(\theta, x) d x d \theta,\right. \\
\left.\int_{r}^{0} \int_{0}^{\ell} D \alpha_{k-1}(\eta(\theta, x))(\tilde{f}(\eta) \otimes \iota)(\theta, x) \beta_{k-1}(\theta, x) d x d \theta, \int_{0}^{\ell}(\tilde{f}(\eta) \otimes \iota)(0, x) d x\right) \\
+\frac{1}{2} \sum_{n=1}^{\infty} \sigma_{n}^{2} D^{2} h(m(\eta))\left\langle\overline{D m(\eta)} \phi_{n} \otimes \iota, \overline{D m(\eta)} \phi_{n} \otimes \iota\right\rangle \\
\cdot D h(m(\eta))\left(\int_{r}^{0} \int_{0}^{\ell} \overline{D^{2} \alpha_{1}(\eta(\theta, x))\left\langle\phi_{n} \otimes \iota, \phi_{n} \otimes \iota\right\rangle(\theta, x) \beta_{1}(\theta, x) d x d \theta,}\right. \\
\ldots \int_{r}^{0} \int_{0}^{\ell} \frac{D^{2} \alpha_{k-1}(\eta(\theta, x))\left\langle\phi_{n} \otimes \iota, \phi_{n} \otimes \iota\right\rangle(\theta, x) \beta_{k}-1}{(\theta, x) d x d \theta, 0\rangle .}
\end{gathered}
$$

Proof: To prove $\mathbb{Q} \mathbb{C} \subset D(\mathcal{A})$, we need to show that each $\Psi=h \circ m \in \mathbb{Q} \mathbb{T}$ satisfies conditions 
$(C 1)-(C 4)$ of $\S 4$. It is clear that each $\Psi \in \mathbb{Q} \mathbb{T}$ is also in $D(\mathcal{A})$. By applying the chain rule under the integral sign, one gets

$$
\begin{aligned}
& D \Psi(\eta)(\xi)=D h(m(\eta)) \cdot\left(\int _ { - r } ^ { 0 } \int _ { 0 } ^ { \ell } D \alpha _ { 1 } ( \eta ( \theta , x ) ) \left(\xi(\theta, x) \beta_{1}(\theta, x) d x d \theta\right.\right. \\
& \left.\ldots, \int_{-r}^{0} \int_{0}^{\ell} D \alpha_{k-1}(\eta(\theta, x))(\xi(\theta, x)) \beta_{k-1}(\theta, x) d x d \theta, \int_{0}^{\ell} \xi(0, x) d x\right),
\end{aligned}
$$

and

$$
\begin{gathered}
D^{2} \Psi(\eta)\left\langle\xi_{1}, \xi_{2}\right\rangle=D^{2} h(m(\eta))\left\langle D m(\eta)\left(\xi_{1}\right), D m(\eta)\left(\xi_{2}\right)\right\rangle \\
+D h(m(\eta))\left(\int_{-r}^{0} \int_{0}^{\ell} D^{2} \alpha_{1}(\eta(\theta, x))\left\langle\xi_{1}(\theta, x), \xi_{2}(\theta, x)\right\rangle \cdot \beta_{1}(\theta, x) d x d \theta,\right. \\
\left.\ldots, \int_{-r}^{0} \int_{0}^{\ell} D^{2} \alpha_{k-1}(\eta(\theta, x))\left\langle\xi_{1}(\theta, x), \xi_{2}(\theta, x)\right\rangle \cdot \beta_{k-1}(\theta, x) d x d \theta, 0\right) .
\end{gathered}
$$

Furthermore, from Theorem 3.4 and Theorem 4.1, we have

$$
\mathcal{A}(\Psi)(\eta)=\varphi(\Psi)(\eta)+D \Psi(\eta)(\tilde{f}(\eta) \otimes \iota)+\frac{1}{2} \sum_{n=1}^{\infty} \sigma_{n}^{2} \overline{D^{2} \Psi(\eta)}\left\langle\phi_{n} \otimes \iota, \phi_{n} \otimes \iota\right\rangle .
$$

The conclusion of the theorem follows.

\section{References}

[1] Bandle, C. and Dozzi, M., Comparison theorems for stochastic differential inequalities and an application to reaction-diffusion equations with random sources, Stoch. Anal. Appl., 12:1 (1994), 11-29.

[2] Busenberg, S. and Huang, W., Stability and Hopf bifurcation for a population delay model with diffusion, J. Diff. Eqns. (to appear).

[3] DaPrato, G. and Zabczyk, J., Stochastic Equations in Infinite Dimensions, Encyclopedia of Mathematics and its Application, 44, Cambridge University Press, New York 1992.

[4] Dynkin, E.B., Markov Processes, Vol. I \& II, Springer-Verlag, Berlin 1965.

[5] Gopalsamy, K., He, X. and Sun, D., Oscillation and convergence in a diffusive delay logistic equation, Math. Nachr., 164 (1993), 219-237.

[6] Gyöngy, I. and Pardoux, E., On quasi-linear stochastic partial differential equations, Prob. Theory Rel. Fields, 94 (1993), 413-425.

[7] Gyöngy, I. and Pardoux, E., On the regularization effect of space-time white noise on quasi-linear parabolic partial differential equations, Prob. Theory Rel. Fields, 97 (1993), 211-229.

[8] Lang, S., Differentiable Manifolds, Addison-Wesley, Reading, MA 1972.

[9] Manthey, R. and Mittmann, K., Bounded solutions of stochastic reaction-diffusion equations, Stoch. and Stoch. Rep., 20:2 (1994), 153-166.

[10] Memory, M.C., Bifurcation and asymptotic behavior of solutions of a delay-differential equation with diffusion, SIAM J. Math. Anal., 20 (1989), 533-546.

[11] Mohammed, S-E A., Stochastic Functional Differential Equations, Research Notes in Mathematics 99, Pitman Adv. Publishing Prog., Boston, London, Melbourne 1984.

[12] Sowers, R., Large deviations for a reaction-diffusion equation with non-Gaussian perturbations, Ann. Prob., 20 (1992), 504-537. 
[13] Sowers, Large deviations for the invariant measure of a reaction-diffusion equation with non-Gaussian perturbations, Prob. Theory Rel. Fields, 92 (1992), 393-421.

[14] Travis, C.C. and Webb, G.F., Existence and stability for partial functional differential equations, Tran. Amer. Math. Soc., 200 (1974), 395-418.

[15] Trevis, F., Topological Vector Spaces, Distributions and Kernels, Academic Press, New York 1967.

[16] Walsh, J.B., An introduction to stochastic partial differential equations, In: École d'Été de Probabilités de Saint-Flour, XIV, (Lecture Notes in Math. 1180), Springer-Verlag, Berlin, Heidelberg, New York 1984. 


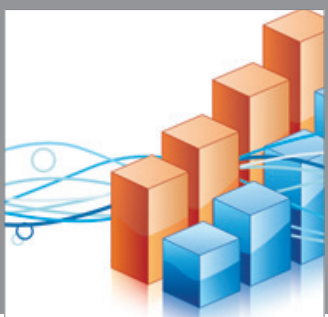

Advances in

Operations Research

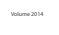

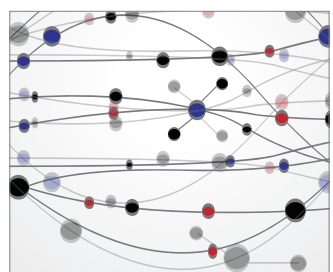

\section{The Scientific} World Journal
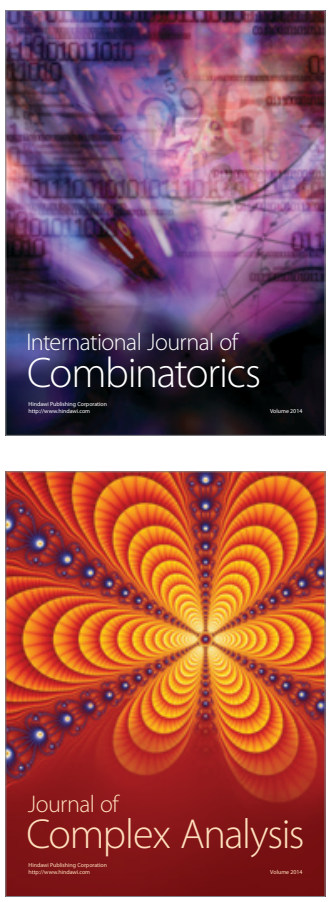

International Journal of

Mathematics and

Mathematical

Sciences
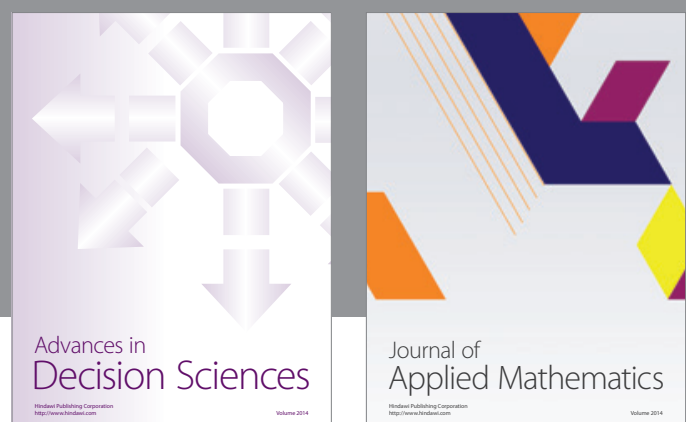

Journal of

Applied Mathematics
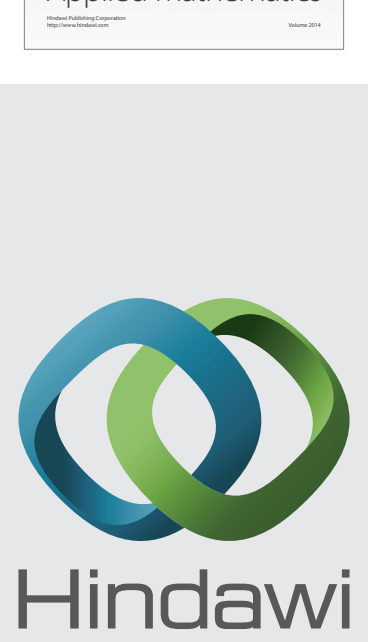

Submit your manuscripts at http://www.hindawi.com
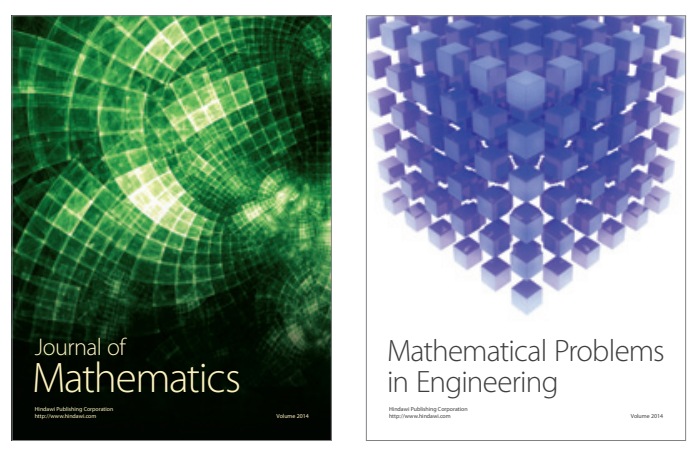

Mathematical Problems in Engineering
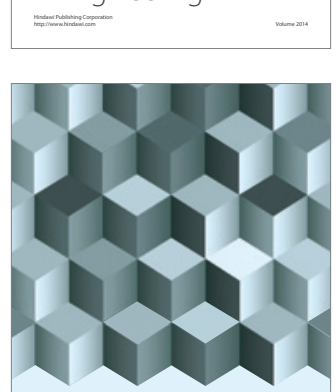

Journal of

Function Spaces
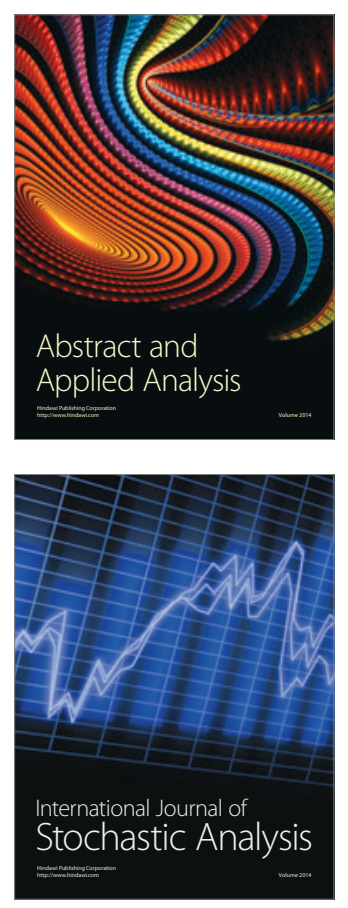

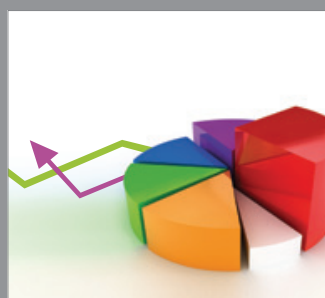

ournal of

Probability and Statistics

Promensencen
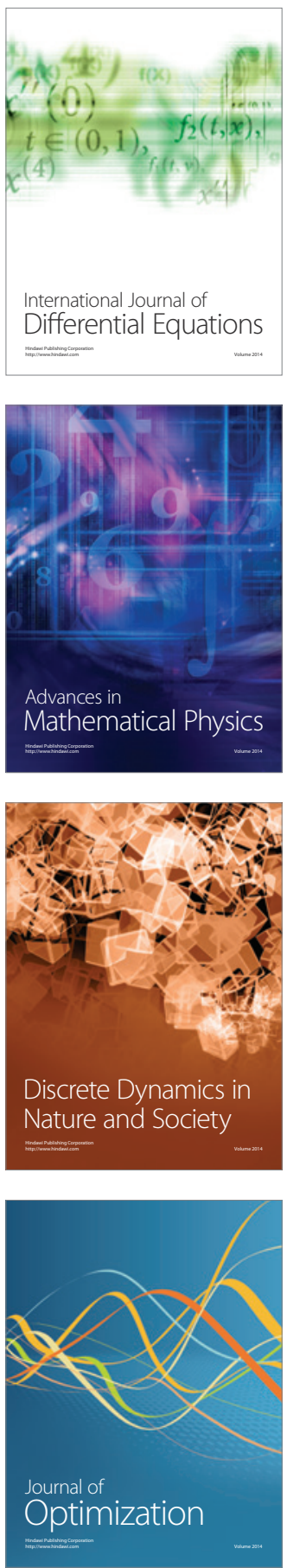